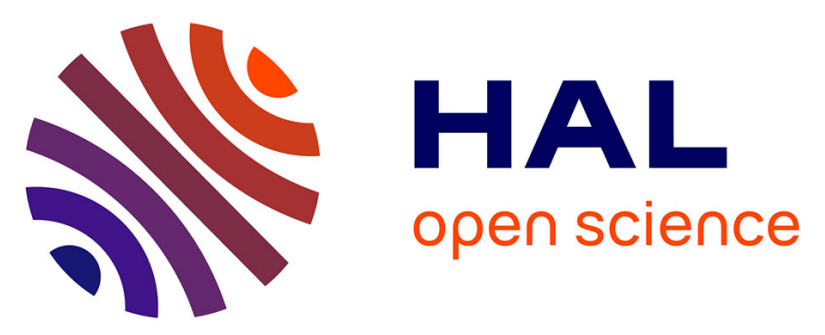

\title{
Reaktionen des metalloiden Clusteranions Ge9[Si(SiMe3)3]3- in der Gasphase. Oxidations- und Reduktionsschritte geben Einblicke in den Bereich zwischen metalloiden Clustern und Zintl-Ionen.
}

Andreas Schnepf, Hansgeorg Schnöckel, Christian Schenk, Florian Henke, Marco Neumaier, Matthias Olzmann

\section{To cite this version:}

Andreas Schnepf, Hansgeorg Schnöckel, Christian Schenk, Florian Henke, Marco Neumaier, et al.. Reaktionen des metalloiden Clusteranions Ge9[Si(SiMe3)3]3- in der Gasphase. Oxidations- und Reduktionsschritte geben Einblicke in den Bereich zwischen metalloiden Clustern und Zintl-Ionen.. Journal of Inorganic and General Chemistry / Zeitschrift für anorganische und allgemeine Chemie, 2010, 636 (7), pp.1173. 10.1002/zaac.201000034 . hal-00552447

\section{HAL Id: hal-00552447 https://hal.science/hal-00552447}

Submitted on 6 Jan 2011

HAL is a multi-disciplinary open access archive for the deposit and dissemination of scientific research documents, whether they are published or not. The documents may come from teaching and research institutions in France or abroad, or from public or private research centers.
L'archive ouverte pluridisciplinaire HAL, est destinée au dépôt et à la diffusion de documents scientifiques de niveau recherche, publiés ou non, émanant des établissements d'enseignement et de recherche français ou étrangers, des laboratoires publics ou privés. 


\section{Reaktionen des metalloiden Clusteranions \{Ge9[Si(SiMe3)3]3\}- in der Gasphase. Oxidations- und Reduktionsschritte geben Einblicke in den Bereich zwischen metalloiden Clustern und Zintl-Ionen.}

\begin{tabular}{|r|l|}
\hline Journal: & Zeitschrift für Anorganische und Allgemeine Chemie \\
\hline Manuscript ID: & zaac. 201000034.R1 \\
\hline Date Submitted by the \\
Author: & 11 -Feb-2010 \\
\hline Complete List of Authors: & $\begin{array}{l}\text { Schnepf, Andreas; University, Inorganic Chemistry } \\
\text { Schnöckel, Hansgeorg } \\
\text { Schenk, Christian } \\
\text { Henke, Florian } \\
\text { Neumaier, Marco } \\
\text { Olzmann, Matthias }\end{array}$ \\
\hline Keywords: & $\begin{array}{l}\text { metalloid cluster compounds, gas phase reactions, germanium, } \\
\text { mass spectrometry, DFT calculations }\end{array}$ \\
\hline \multicolumn{2}{|c|}{} \\
\hline
\end{tabular}

\section{今 scholarONE" \\ Manuscript Central}




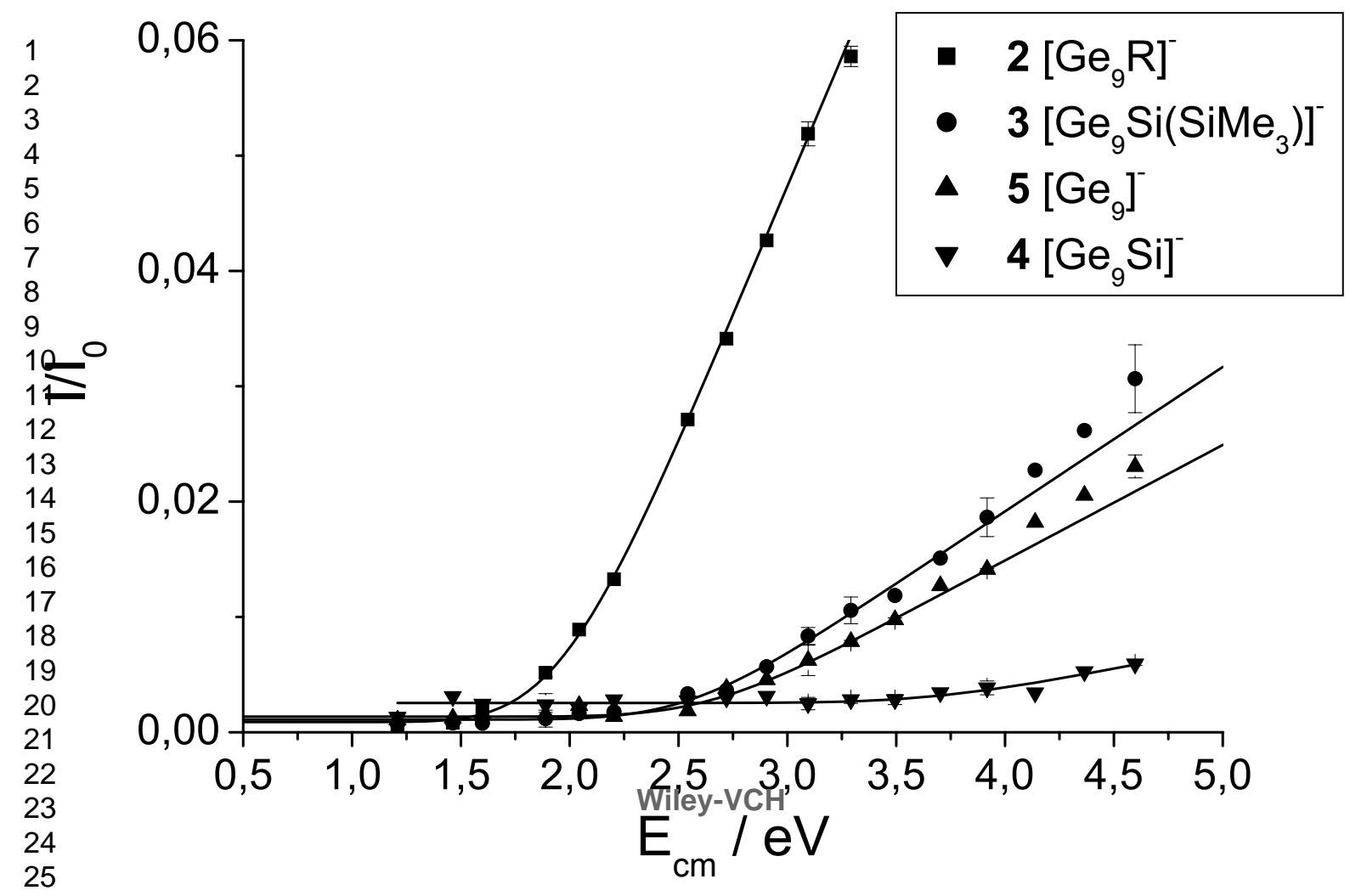




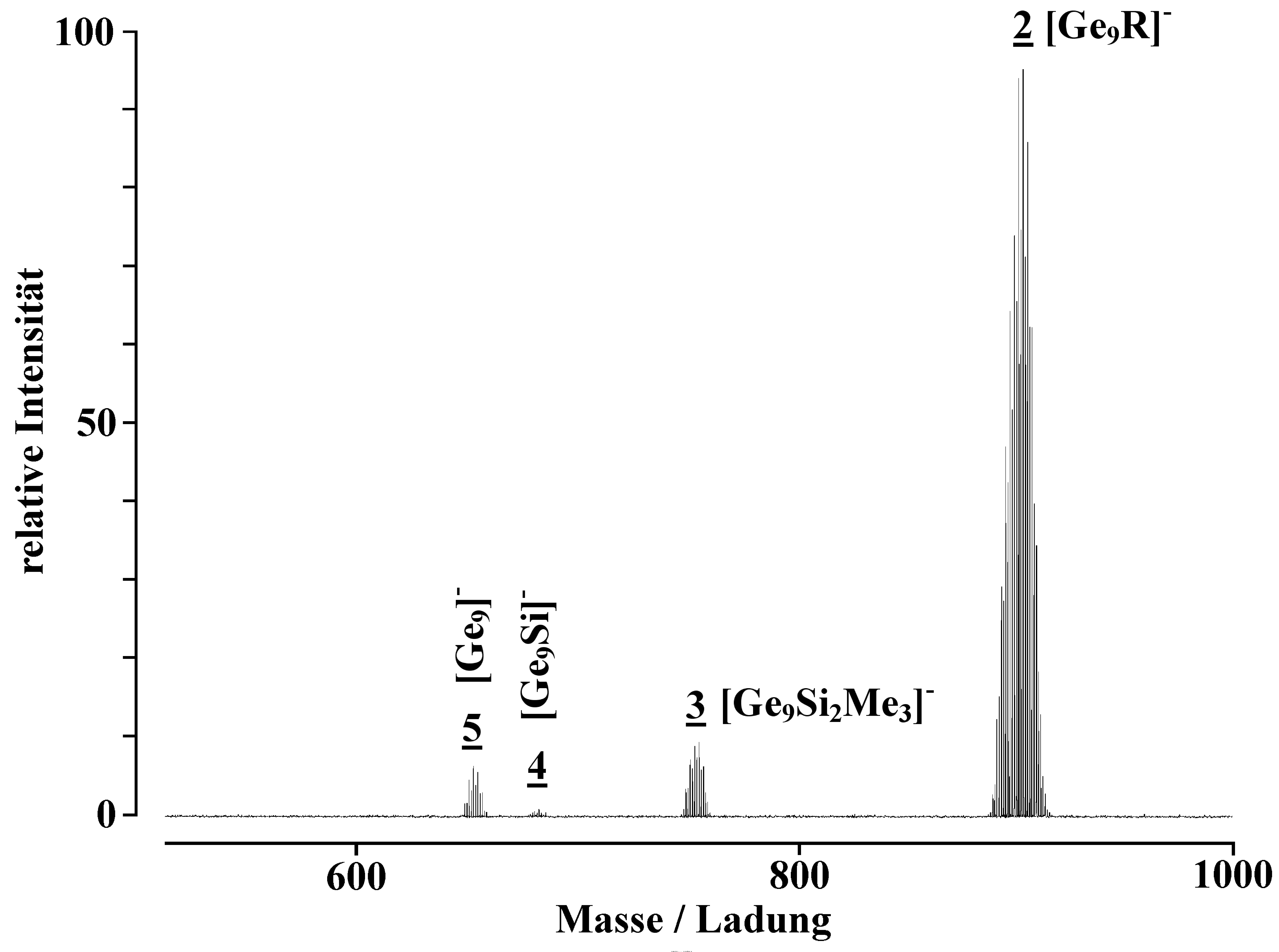




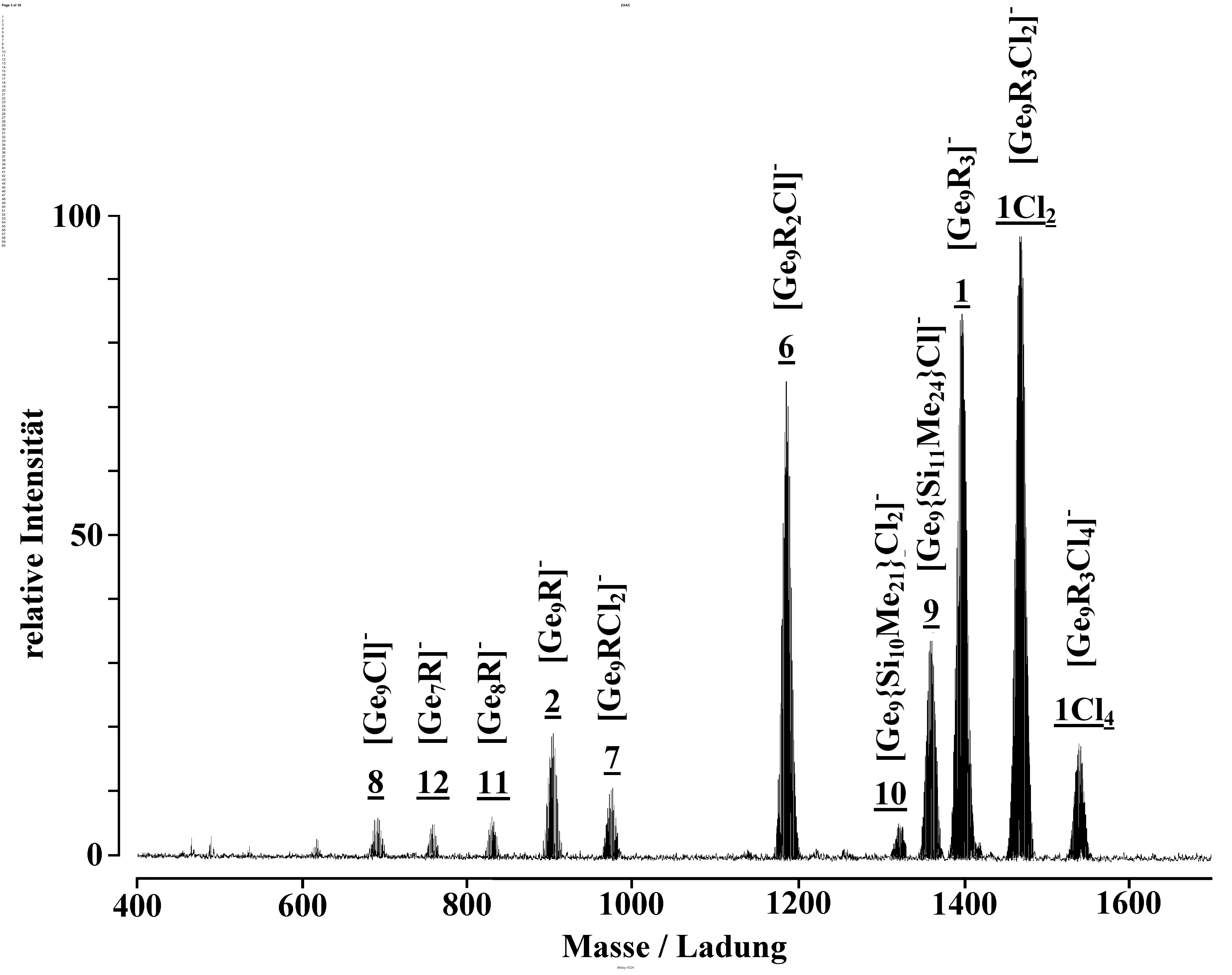




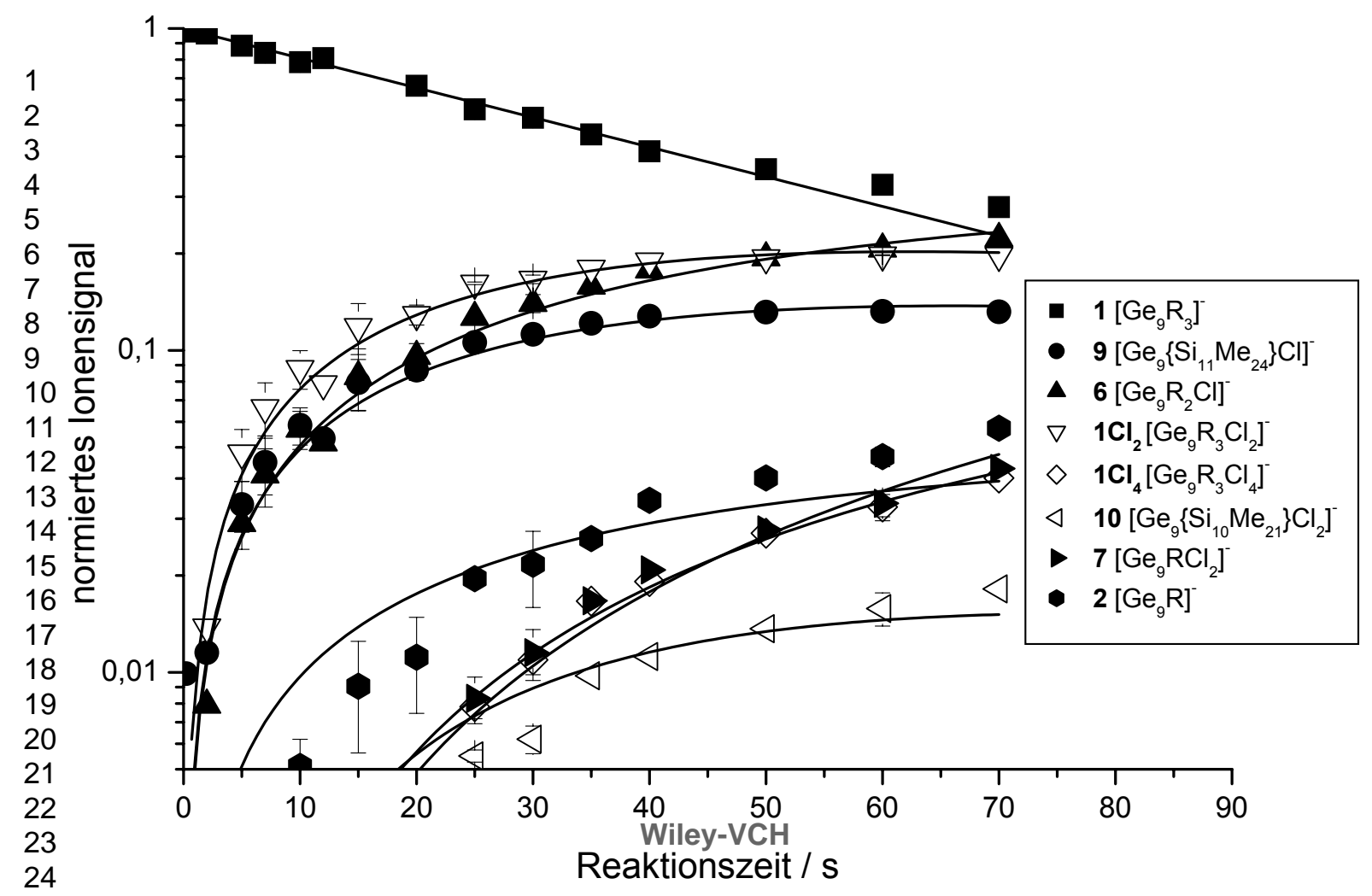

25 
ZAAC

$\longrightarrow \quad\left[\mathrm{Ge}_{9} \mathrm{R}_{3} \mathrm{Cl}_{2}\right]^{-}$ $\mathbf{1 C l}_{2}$
$+\mathrm{Cl}_{2}$

$\left[\mathrm{Ge}_{9} \mathrm{R}_{3} \mathrm{Cl}_{4}\right]^{-}$ $\mathbf{1 C l}_{4}$

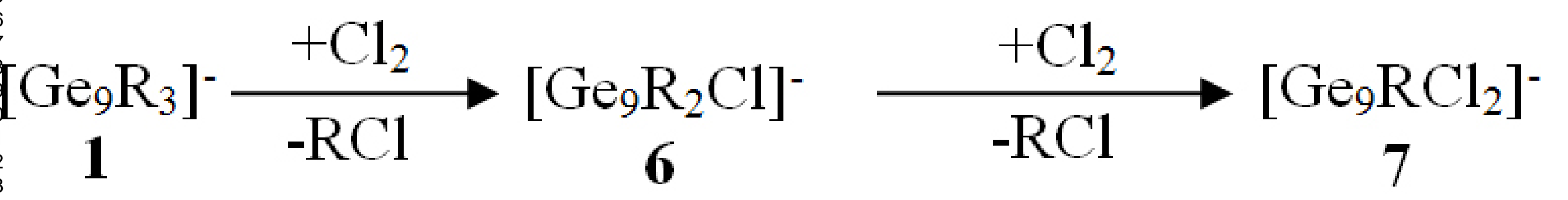
-

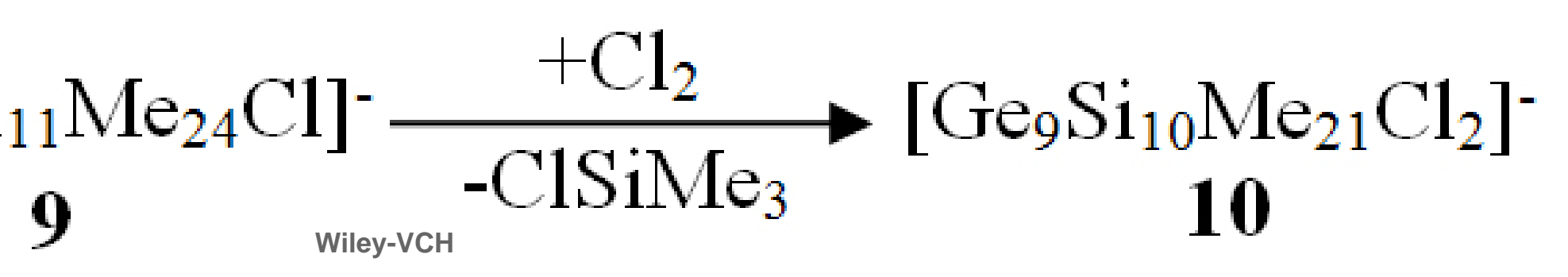



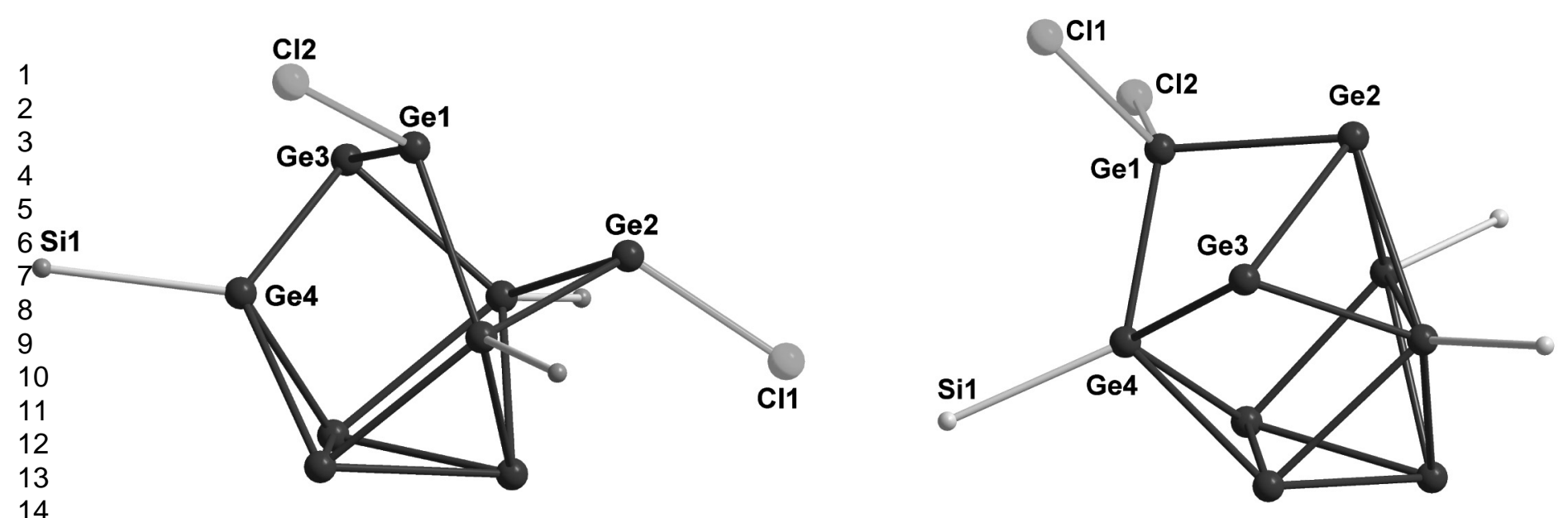
$\odot$

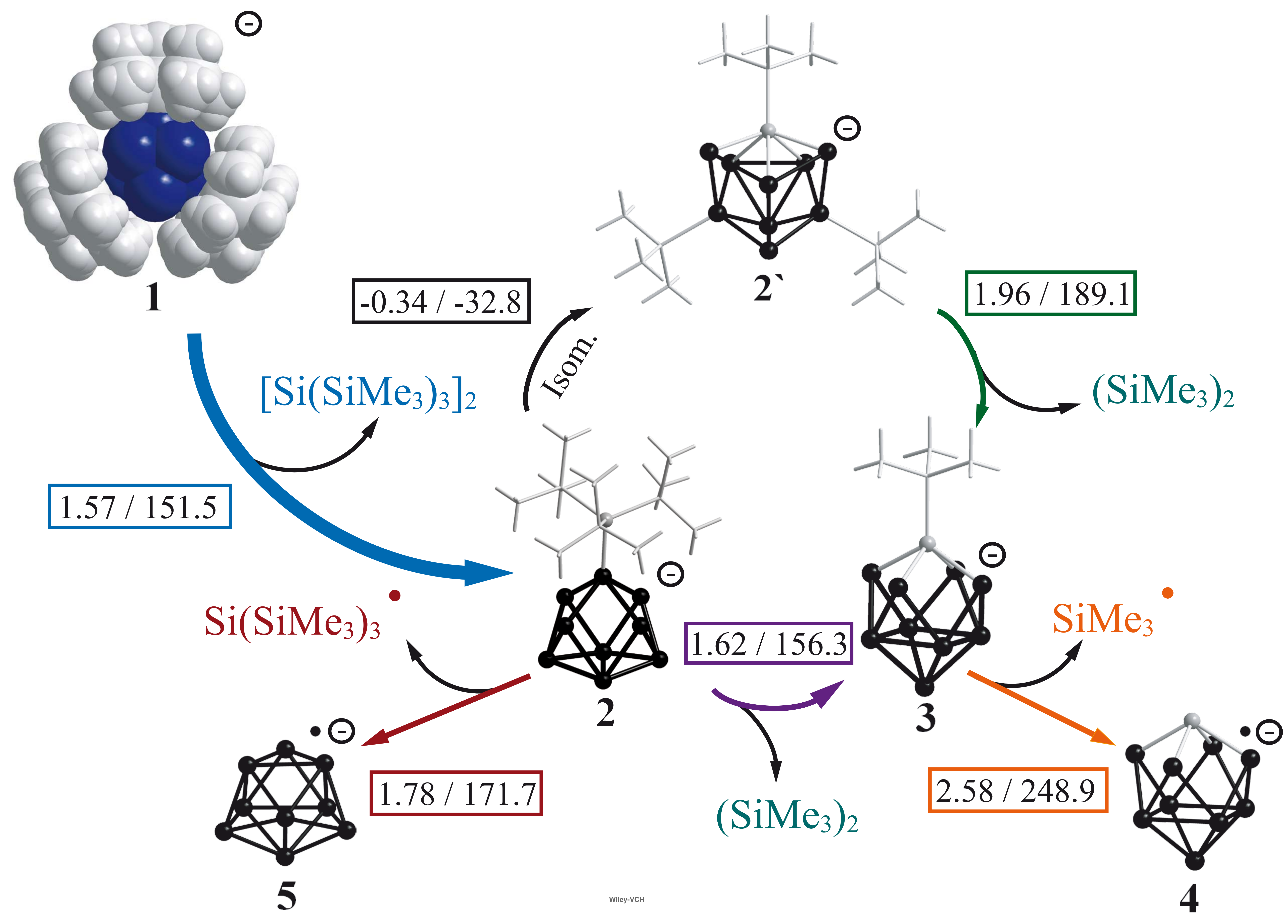




\title{
Reaktionen des metalloiden Clusteranions $\left\{\mathrm{Ge}_{9}\left[\mathrm{Si}\left(\mathrm{SiMe}_{3}\right)_{3}\right]_{3}\right\}^{-}$in der Gasphase. Oxidations- und Reduktionsschritte geben Einblicke in den Bereich zwischen metalloiden Clustern und Zintl-Ionen.
}

\author{
Christian Schenk, Florian Henke, Marco Neumaier, Matthias Olzmann ${ }^{\mathrm{a}}$, Hansgeorg \\ Schnöckel* und Andreas Schnepf*
}

Karlsruher Institut für Technologie (KIT) Institut für Anorganische Chemie
${ }^{a}$ Karlsruher Institut für Technologie (KIT) Institut für Physikalische Chemie

Professor Gerd Becker zum 70. Geburtstag gewidmet.

Bei der Redaktion eingegangen am:

\author{
Reactions of the metalloid cluster anion $\left\{\mathrm{Ge}_{9}\left[\mathrm{Si}\left(\mathrm{SiMe}_{3}\right)_{3}\right]_{3}\right\}^{-}$in the gas phase. \\ Oxidation- and reduction-steps give an insight onto the area between metalloid clusters \\ and Zintl ions
}
Keywords: metalloid cluster compounds, Germanium, gas phase reactions, DFT calculations, mass spectrometry, Zintl ions

*Prof. Dr. H. Schnöckel, PD Dr. A. Schnepf

Karlsruher Institut für Technologie (KIT) Institut für Anorganische Chemie

Engesserstraße 15, Geb. 30.45, D-76131 Karlsruhe

Tel.: +49 (0)721 608-2951; Fax: +49 (0)721 608-4854

Email: schnepf@chemie.uni-karlsruhe.de 


\begin{abstract}
The cluster anion $\left\{\mathrm{Ge}_{9}\left[\mathrm{Si}\left(\mathrm{SiMe}_{3}\right)_{3}\right]_{3}\right\}^{-} \mathbf{1}$ is transferred intact into the gas phase via the electro spray method. Subsequently the fragmentation of $\mathbf{1}$ after resonant excitation as well as the oxidation reaction with $\mathrm{O}_{2}$ and $\mathrm{Cl}_{2}$ are investigated in an FT-ICR mass spectrometer (Fourier transform ion cyclotron). Unlike former results with off-resonant excitation the fragmentation leads mainly to the end-product $\mathrm{Ge}_{9}^{-}$. Moreover, applying an on-resonant excitation the dissociation experiment can be quantified; $2.0 \pm 0.15 \mathrm{eV}(193 \pm 15 \mathrm{~kJ} / \mathrm{mol})$ for the elimination of the first two ligands and $2.7 \pm 0.15 \mathrm{eV}(261 \pm 15 \mathrm{~kJ} / \mathrm{mol})$ for all ligands, respectively. Particular attention is turned on the first step, where sterically encumbered $\mathrm{Si}_{2}\left(\mathrm{SiMe}_{3}\right)_{6}$ molecules are formed in a concerted reaction. This result, which is also important for elemental reactions on metal surfaces in catalyses, is based on experimentally determined threshold energies, DFT calculations and calculations on the lifetime of the involved species. In contrast to the high reactivity of crystalline $1 \cdot \mathrm{Li}(\mathrm{THF})_{4}$, gaseous $\mathbf{1}$ is inert against oxygen. The analogy to recently published spin forbidden reactions of $\mathrm{Al}_{13}{ }^{-}$with $\mathrm{O}_{2}$ hints to a general importance of spin conversion during gas phase reactions of larger cluster molecules. The oxidation of 1 with $\mathrm{Cl}_{2}$ proceeds via different reaction channels. DFT calculations give a first insight on the complex primary oxidation steps. These calculations also reveal that the delocalized bonding situation in the $\mathrm{Ge}_{9}$ core is distorted upon oxidation. This result together with the dissociation experiments shed more light on differences and similarities between metalloid clusters and Zintl ions.
\end{abstract}

\title{
Kurztext
}

Das Clusteranion $\left\{\mathrm{Ge}_{9}\left[\mathrm{Si}\left(\mathrm{SiMe}_{3}\right)_{3}\right]_{3}\right\}^{-} \mathbf{1}$ wird mithilfe der Elektrospraymethode unzersetzt in die Gasphase überführt. Anschließend werden in einem FT-ICR-Massenspektrometer 
(Fourier-Transformation-Ionen-Cyclotron) seine Fragmentierung nach resonanter Anregung und sein Oxidationsverhalten gegenüber $\mathrm{O}_{2}$ und $\mathrm{Cl}_{2}$ untersucht. Die Fragmentierungsversuche ergeben, anders als bei früheren off-resonanten Untersuchungen, hauptsächlich das Endprodukt $\mathrm{Ge}_{9}{ }^{-}$. Außerdem lassen sich mit der hier verwendeten on-resonanten Technik die Dissoziationsenergien für die Abspaltung der ersten beiden Liganden mit 2.0 $\pm 0.15 \mathrm{eV}$ $(193 \pm 15 \mathrm{~kJ} / \mathrm{mol})$ und des letzten Liganden mit $2.7 \pm 0.15 \mathrm{eV}(261 \pm 15 \mathrm{~kJ} / \mathrm{mol})$ bestimmen. Besonderes Augenmerk gilt der Untersuchung des ersten Schrittes, bei dem in einer konzertierten Reaktion das sterisch überfrachtete $\mathrm{Si}_{2}\left(\mathrm{SiMe}_{3}\right)_{6}$ entsteht. Dieser Befund, dem unter anderem auch für Elementarreaktionen bei der Reaktion auf Metalloberflächen in der Katalyse Bedeutung zukommt, resultiert aus der experimentellen Bestimmung der Schwellenenergie, begleitenden DFT Rechnungen und Berechnungen zur Lebensdauer der beteiligten Spezies.

Gegenüber elementarem Sauerstoff ist gasförmiges 1 im Gegensatz zur kristallinen Clusterverbindung $\mathbf{1} \cdot \mathrm{Li}(\mathrm{THF})_{4}$ inert. Die Analogie zu den jüngst publizierten spinverbotenen Reaktionen zwischen $\mathrm{Al}_{13}{ }^{-}$und $\mathrm{O}_{2}$ weist auf die generelle Bedeutung des Spinerhaltungssatzes bei Gasphasenreaktionen größerer Clustermoleküle hin. Die spontane Oxidation von 1 mit $\mathrm{Cl}_{2}$ verläuft über unterschiedliche Reaktionskanäle. DFT Rechnungen erlauben einen ersten Einblick in die komplexen primären Oxidationsschritte. Außerdem zeigen diese orientierenden Rechnungen, dass die delokalisierte Bindungssituation im $\mathrm{Ge}_{9}$-Gerüst durch die Oxidation zerstört wird. Dieser Befund und die Fragmentierungsexperimente erlauben es, die Unterschiede und Gemeinsamkeiten zwischen metalloiden Clustern und Zintl-Ionen näher zu beleuchten.

\section{Einleitung}

Die ersten massenspektrometrischen Untersuchungen zur Fragmentierung und zu Reaktionen eines metalloiden Clusters wurden kürzlich für das Clusteranion $\mathrm{Ga}_{19}\left[\mathrm{C}\left(\mathrm{SiMe}_{3}\right)_{3}\right]_{6}{ }^{-}$ 
beschrieben $\left(\mathrm{Ga}_{19}\left[\mathrm{C}\left(\mathrm{SiMe}_{3}\right)_{3}\right]_{6}{ }^{-} \rightarrow \mathrm{Ga}_{13}{ }^{-}+6 \mathrm{GaC}\left(\mathrm{SiMe}_{3}\right)_{3} ; \mathrm{Ga}_{19}\left[\mathrm{C}\left(\mathrm{SiMe}_{3}\right)_{3}\right]_{6}{ }^{-}+\mathrm{Cl}_{2} \rightarrow \ldots\right){ }^{1}$

Diese Experimente waren der Schlüssel zum Verständnis der Bindungssituation in metalloiden Clustern der XIII. Gruppe. Basierend auf diesen Ergebnissen haben wir für einen metalloiden Cluster der XIV. Gruppe vor kurzem orientierende Fragmentierungsversuche mit

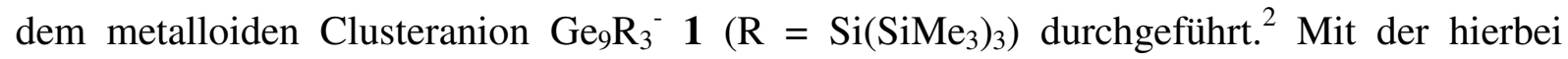
verwendeten SORI-CAD Methode ${ }^{1 \mathrm{a}, 3}$ (sustained off-resonance irradiation collisional activation dissociation) gelang es, die Fragmentierung unter Bruch der jeweils schwächsten Bindungen von einzelnen angeregten $\mathrm{Ge}_{9} \mathrm{R}_{3}$-Anionen $\mathbf{1}$ nach Stößen mit Inertgasmolekülen zu beobachten. Die wichtigsten Ergebnisse dieser Untersuchung waren: a.) im Gegensatz zu den Experimenten bei $\mathrm{Ga}_{19} \mathrm{R}_{6}{ }^{-}$Clustern werden keine Metall-Metall Bindungen gebrochen; $\mathrm{b}$.) die ersten beiden Liganden $\mathrm{R}$ werden nicht nacheinander als Radikale, sondern in unerwarteter Weise in einem Schritt abgespalten und c.) als Endprodukte der Ligandenfragmentierung erhält man im wesentlichen $\mathrm{Ge}_{9} \mathrm{Si}$-Clusteranionen, d.h. es hat eine Clustererweiterung stattgefunden. Diese orientierenden Versuche, bei denen noch viele Fragen offen blieben, haben wir in der jetzt vorgelegten Arbeit aufgegriffen und durch on-resonante Anregungsversuche zu 1 vertieft und zum Teil quantifiziert. Solche Ergebnisse fehlten für das Verständnis der neuartigen Bindungsverhältnisse im Gebiet der metalloiden Germaniumcluster, ${ }^{4}$ das sich in den letzten Jahren rasant entwickelt hat und in dem $\mathbf{1}$ auch hinsichtlich seines Synthesepotentials eine Sonderstellung einnimmt. ${ }^{5,6}$

Zur kinetischen Stabilisierung einer Reihe von Verbindungen dieser neuen Klasse von Germaniumclustern, die als Modelle für den Grenzbereich zwischen Molekülen und Festkörpern gelten, ${ }^{7}$ werden meist sterisch anspruchsvolle Liganden wie $\mathrm{Si}\left(\mathrm{SiMe}_{3}\right)_{3}$, $\mathrm{N}\left(\mathrm{SiMe}_{3}\right)_{2}, 2,6-\mathrm{Ar}_{2}-\mathrm{C}_{6} \mathrm{H}_{3}\left(\mathrm{Ar}=2,6-i \mathrm{Pr}_{2}-\mathrm{C}_{6} \mathrm{H}_{3}\right)$ u.s.w. verwendet. ${ }^{8}$ Während diese Liganden in den meisten Fällen, der gewünschten Stabilisierungsintention entsprechend, den Clusterkern fast völlig abschirmen, sodass Folgereaktionen am Kerngerüst kaum möglich sind, liegen die Verhältnisse für $\left\{\mathrm{Ge}_{9}\left[\mathrm{Si}\left(\mathrm{SiMe}_{3}\right)_{3}\right]_{3}\right\}^{-} \mathbf{1}^{9}$ völlig anders (siehe Kalottenmodell Schema 1): Hier 
lassen sich z.B. die nackten Germaniumatome in $\mathbf{1}$ durch Umsetzung mit Übergangsmetallkationen $\mathrm{M}^{\mathrm{n}+}$ zu neutralen und ionischen $\mathrm{MGe}_{18}\left[\mathrm{Si}\left(\mathrm{SiMe}_{3}\right)_{3}\right]_{6}$-Verbindungen absättigen. ${ }^{5}$ Die hohe Reaktivität dieser ungeschützten $\mathrm{Ge}_{9} \mathrm{R}_{3}{ }^{-}$-Cluster wird neben Reaktionen mit ungesättigten Komplexverbindungen ${ }^{6}$ auch durch die spontane oxidative Zersetzung der Clusterverbindung $\mathbf{1} \cdot \operatorname{Li}(\text { thf })_{4}$ bei Luftkontakt deutlich.

Um einen ersten Einblick in den Mechanismus solcher oxidativen Zersetzungen von $\mathbf{1}$ zu erhalten, haben wir die Gasphasenreaktionen von $\mathbf{1}$ mit $\mathrm{Cl}_{2}$ und $\mathrm{O}_{2}$ massenspektrometrisch untersucht. Diese Ergebnisse und die oben genannten energieaufgelösten Experimente bei stoßinduzierten Dissoziationen erlauben erstmals quantitative Einblicke in die Bindungsverhältnisse und die komplexen oxidativen $\left(\mathrm{Cl}_{2}\right.$ bzw. $\left.\mathrm{O}_{2}\right)$ und reduktiven $\left(\mathrm{R}_{2}\right.$ Eliminierung) Abbauprozesse eines metalloiden Ge-Clusters. Die im folgenden präsentierten experimentellen Ergebnisse von $\mathbf{1}$ in der ICR-Ionenfalle eines FT-ICR-Massenspektrometers (Fourier-Transfomation-Ionen-Cyclotron) bei ca. $10^{-10} \mathrm{mbar}$ - diese Bedingungen sind für die Untersuchung von Einzelstößen zwischen Molekülen notwendig - werden durch DFTRechnungen und durch orientierende, zum Teil auch quantitative Untersuchungen zur Kinetik dieser Reaktionen ergänzt.

\section{Ergebnisse und Diskussion}

Um das Verständnis der Bindungssituation in $\mathrm{Ge}_{9} \mathrm{R}_{3}^{-} \mathbf{1} \mathrm{zu}$ vertiefen, beschreiben und charakterisieren wir zunächst die Fragmentierung. Dabei soll unter anderem geklärt werden, wie die sowohl bei früheren massenspektrometrischen Experimenten, als auch bei Synthesearbeiten beobachtete Abspaltung von $\mathrm{R}_{2}$ Molekülen $\left(\mathrm{Ge}_{9} \mathrm{R}_{3}{ }^{-} \rightarrow \mathrm{Ge}_{9} \mathrm{R}^{-}+\mathrm{R}_{2}\right)$ unter Einzelstoßbedingungen im Detail abläuft. Anschließend wird nach der Beschreibung der unerwarteten Inertheit von 1 gegenüber $\mathrm{O}_{2}$ über die Oxidationsschritte von 1 mit $\mathrm{Cl}_{2}$ berichtet. 


\section{Fragmentierungsversuche}

Wie wir bereits früher detailliert beschrieben haben, ${ }^{2}$ lässt sich das Clusteranion $\mathrm{Ge}_{9} \mathrm{R}_{3}{ }^{-} \mathbf{1}[\mathrm{R}$ $\left.=\mathrm{Si}\left(\mathrm{SiMe}_{3}\right)_{3}\right]$ aus einer THF Lösung mithilfe der Elektrospray-Ionisationsmethode $(\mathrm{ESI})^{10}$ unzersetzt in die Gasphase überführen und anschließend in die ICR-Ionenfalle eines FT-ICRMassenspektrometers transferieren. Dort wird bei den hier beschriebenen Versuchen die kinetische Energie von 1 durch resonante dipolare Anregung variabel erhöht. Wird nun gleichzeitig über ein Leckventil ein statischer Argonpartialdruck (ca. $2 \cdot 10^{-8}$ mbar) eingestellt, so lässt sich durch Kollision mit dem Stoßgas (Argon) ein Teil der kinetischen Energie von 1 in innere Energie überführen, bis es zur Fragmentation kommt. ${ }^{11,12}$ Erhöht man die kinetische Energie von 1 schrittweise, so führen die Stöße mit den Argonatomen bereits bei $2.0 \pm 0.15 \mathrm{eV}$ $(193 \pm 15 \mathrm{~kJ} / \mathrm{mol})$ zu einer Eliminierung von zwei $\mathrm{Si}\left(\mathrm{SiMe}_{3}\right)_{3}$ Liganden und zur Bildung von $\mathrm{Ge}_{9} \mathrm{R}^{-} 2$ (Schema 1). Die weitere Erhöhung der durch Stöße übertragenen inneren Energie von

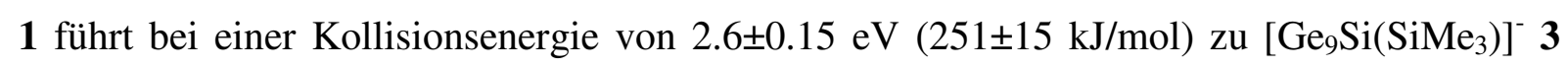
und bei $2.7 \pm 0.15 \mathrm{eV}(261 \pm 15 \mathrm{~kJ} / \mathrm{mol}) \mathrm{zu} \mathrm{Ge}_{9}{ }^{-} 5$ (Schema 1). Erst ab einer Energie von etwa $3.8 \mathrm{eV}(367 \mathrm{~kJ} / \mathrm{mol})$ kommt es zur Bildung von $\mathrm{Ge}_{9} \mathrm{Si}^{-}$4. Die angegebenen Stoßenergiewerte resultieren aus den gemessenen Schwellwertkurven, die in Abbildung 1 wiedergegeben sind.

$<<$ Schema 1 hier $>>>>$

Schema 1: Kalottenmodell von $\left\{\mathrm{Ge}_{9}\left[\mathrm{Si}\left(\mathrm{SiMe}_{3}\right)_{3}\right]_{3}\right\}^{-} \mathbf{1}$ (Blick entlang der dreizähligen Achse) und Verlauf der stoßinduzierten Dissoziation von 1 mit „off-,, und „on-resonanter“ Anregung (siehe Text); die angegebenen Energiewerte (eV, kJ/mol) stammen aus DFT Rechnungen. Die angegebenen Molekülstrukturen von 2, 2, 3 - 5 sind durch DFT Rechnungen ermittelte Minimumstrukturen. 
Diese quantitativen Ergebnisse der Fragmentierung von 1 unterscheiden sich von den früheren qualitativen Ergebnissen an 1, bei denen die Schwellenwerte für die einzelnen Dissoziationsstufen nicht zugänglich waren, da sie mit einer off-resonanten Anregung (SORICAD; sustained off-resonance irradiation collisional activation dissociation) erfolgten. Bei dieser SORI-CAD-Methode wird in dem zu fragmentierenden Molekül durch Stöße z.B. mit Argonatomen nach und nach so viel Energie akkumuliert bis es zum Bruch der jeweils schwächsten Bindung kommt. Dabei fragmentiert 1, wie bei den hier beschriebenen resonanten Anregungsversuchen, zunächst zu 2 (Schema 1). Danach kommt es jedoch bei offresonanter Anregung aufgrund des unterschiedlichen Anregungsmechanismus (s.o.) zu einer Isomerisierung zu 2', dass anschließend $\left(\mathrm{SiMe}_{3}\right)_{2}$ eliminiert und $\mathrm{zu} \mathbf{3}$ fragmentiert. ${ }^{13}$ Das Endprodukt dieses Fragmentierungskanals ist fast ausschließlich $\mathrm{Ge}_{9} \mathrm{Si}^{-} 4$ (Schema 1), d.h. es besteht nicht nur ein quantitativer sondern auch ein prinzipieller Unterschied zu den hier beschriebenen Ergebnissen. ${ }^{14}$

$<<$ Abbildung 1 hier $>>>$

Abbildung 1: Schwellwertkurve von $\left[\mathrm{Ge}_{9} \mathrm{R}_{3}\right]^{-} 1$ nach on-resonanter Anregung und Kollision mit Argon $\left(\mathrm{t}_{\mathrm{diss}}=100 \mathrm{~ms}, \mathrm{p}=2.2 \cdot 10^{-8}\right.$ mbar $)$ als Funktion der Stoßenergie $\mathrm{E}_{\mathrm{cm}}(1 \mathrm{eV}=96.5$ $\mathrm{kJ} / \mathrm{mol}$ ). Die durchgezogenen Kurven resultieren aus einer Anpassung an die gemessenen Datenpunkte. Bei $\mathbf{3}$ und $\mathbf{4}$ wurden jeweils nur die Datenpunkte bis $3.9 \mathrm{eV}$ angepasst.

Um das unterschiedliche Fragmentierungsverhalten von 1 im zweiten Schritt $\left(\mathrm{Ge}_{9} \mathrm{R}^{-} \rightarrow \ldots\right)$ bei der hier beschriebenen resonanten Anregung gegenüber früheren SORI-CAD Experimenten $\mathrm{zu}$ verstehen, d.h. um den Fortgang des Fragmentierungsmechanismus aufzuklären, haben wir das bei beiden Verfahren entstehende Primärprodukt, also das Clusteranion $\mathrm{Ge}_{9} \mathrm{R}^{-} \mathbf{2}$ in möglichst großen Konzentrationen erzeugt und als einziges Ion 
isoliert. ${ }^{15,16}$ Anschließend wurde 2 resonant angeregt und für $100 \mathrm{~ms}$ mit Argonatomen zur Kollision gebracht (s.o.). Bei Schwellenwerten von ca. 2 eV (Schwellwertkurven siehe Zusatzinformationen Abbildung Z1) entstehen die Clusteranionen $\left[\left(\mathrm{Ge}_{9} \mathrm{Si}\right) \mathrm{SiMe}_{3}\right]^{-} \mathbf{3}$ und $\mathrm{Ge}_{9}{ }^{-}$ 5, die im Spektrum (Abbildung 2) deutlich zu erkennen sind. Bei resonanter Anregung kann 2 also in zwei Fragmentierungskanälen zerfallen: $\mathrm{Ge}_{9}{ }^{-} \mathbf{5}(2.3 \pm 0.15 \mathrm{eV})$ und $\left[\left(\mathrm{Ge}_{9} \mathrm{Si}\right) \mathrm{SiMe}_{3}\right]^{-} 3$ $(2.0 \pm 0.15 \mathrm{eV})$, wobei 3 durch Einzelstöße in dem zugeführten Energiebereich von ca. $2.0 \mathrm{eV}$ nicht $\mathrm{zu} \mathrm{Ge}_{9} \mathrm{Si}^{-} 4$ weiterreagieren können sollte. Die trotzdem beobachtete geringe Konzentration an 4 in Abbildung 2 ist vermutlich darauf zurückzuführen, dass es bei den vorhandenen Versuchsbedingungen ab einer Schwellenenergie von $1.7 \pm 0.2 \mathrm{eV}$ mit einer geringen Wahrscheinlichkeit zu Folgestößen kommen kann, durch die soviel Energie akkumuliert wird, dass bei der Fragmentierung sogar $\mathrm{Ge}_{9} \mathrm{Si}^{-}$Cluster 4 gebildet werden (Zusatzinformationen Abbildung Z1).

$<<<$ Abbildung 2 hier $>>>$

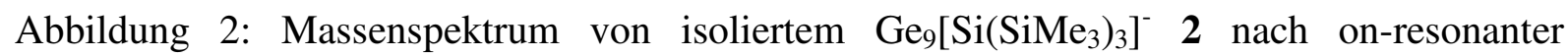
Stoßanregung mit Argonatomen. Den einzelnen Signalgruppen sind die entsprechenden Summenformeln zugeordnet.
\end{abstract}

Sämtliche experimentellen Befunde, die primäre Dissoziation der beiden ersten Liganden $\left(\mathrm{Ge}_{9} \mathrm{R}_{3}^{-} \rightarrow \mathrm{Ge}_{9} \mathrm{R}^{-}+\mathrm{R}_{2}\right)$ bei $2.0 \mathrm{eV}$ und die erschwerte Dissoziation des dritten Liganden $\left(\mathrm{Ge}_{9} \mathrm{R}_{3}{ }^{-} \rightarrow \mathrm{Ge}_{9}{ }^{-}+\mathrm{R}_{2}+\mathrm{R}\right)$ bei ca. $2.7 \mathrm{eV}$ sind in Einklang mit den aus DFT Rechnungen ${ }^{17}$ stammenden thermodynamischen Fragmentierungsenergien (Schema 1). Diese Befunde scheinen plausibel, da im ersten Schritt geschlossenschalige und im weiteren Schritt radikalische Spezies $\left(\mathrm{Ge}_{9}^{-\bullet}\right.$ und $\left.\mathrm{R}^{\bullet}\right)$ entstehen. Da jedoch der erste Schritt auf der „Clusteroberfläche“ die sterisch schwer vorstellbare Dimerisierung von zwei $\operatorname{Si}\left(\mathrm{SiMe}_{3}\right)_{3}$ 
Liganden zu einem $\mathrm{Si}_{2}\left(\mathrm{SiMe}_{3}\right)_{6}$ Molekül und dessen anschließende Abspaltung umfasst, haben wir untersucht, ob dieser sterisch erschwerte Schritt einer genaueren Überprüfung hinsichtlich der Lebensdauer der beteiligten Spezies standhält. Wir haben die folgenden Untersuchungen auch deshalb durchgeführt, da solchen reduktiven Eliminierungsreaktionen auch eine allgemeine Bedeutung zukommt.

Bei sehr großen Ionen kann die beobachtete Dissoziationsschwelle beträchtlich von der tatsächlichen Bindungsenergie abweichen, da unter Umständen sehr viel mehr Energie notwendig ist, um im experimentellen Zeitfenster einen Bindungsbruch zu induzieren. Die Ursache dieses „kinetischen Shifts“18 liegt darin begründet, dass die Stoßenergie bei großen Molekülen auf sehr viele Schwingungsfreiheitsgrade (381 bei $\left.\mathrm{Ge}_{9} \mathrm{R}_{3}{ }^{-} \mathbf{1}\right)$ umverteilt wird und die Wahrscheinlichkeit sinkt, dass die für den Bindungsbruch notwendige Energie in der kritischen, zum Bindungsbruch führenden Schwingungsmode lokalisiert wird.

Mit Hilfe der statistischen Phasenraumtheorie (PST) ${ }^{19}$ lassen sich als Funktion der inneren Energie Lebensdauern der Clusterionen berechnen und die notwendige Energie abschätzen, welche im Cluster dissipiert werden muss, um auf z.B. einer Zeitskala von $100 \mathrm{~ms}$ eine detektierbare Fragmentierung zu erhalten. Im Falle von $\mathrm{Ge}_{9} \mathrm{R}_{3}{ }^{-} 1$ konnte durch entsprechende Berechnungen (zur Methodik siehe [20] und dort zitierte Literatur) gezeigt werden, dass $\mathrm{Ge}_{9} \mathrm{R}_{3}{ }^{-}$bei einer inneren Energie von $\mathrm{E}_{\text {int }}=6.8 \mathrm{eV}$ mit einer Geschwindigkeitskonstanten im Bereich von $0.1 \mathrm{~s}^{-1}$ in die Fragmente $\mathrm{Ge}_{9} \mathrm{R}_{2}{ }^{--}+\mathrm{R}^{\bullet}$ und $\mathrm{Ge}_{9} \mathrm{R}^{-}+\mathrm{R}_{2}$ (Dissoziationsenergien: $2.07 \mathrm{eV}$ bzw. $1.57 \mathrm{eV}$ ) zerfällt und man somit auf einer experimentellen Zeitskala von $100 \mathrm{~ms}$ einen Zerfall in $\mathrm{Ge}_{9} \mathrm{R}_{2}{ }^{--}$und $\mathrm{Ge}_{9} \mathrm{R}^{-}$detektieren sollte (Bildung von $\sim 1 \%\left(\mathrm{Ge}_{9} \mathrm{R}_{2}{ }^{--}+\mathrm{Ge}_{9} \mathrm{R}^{-}\right)$). Das berechnete Verhältnis $\left[\mathrm{Ge}_{9} \mathrm{R}_{2}{ }^{-}\right] /\left[\mathrm{Ge}_{9} \mathrm{R}^{-}\right]$hängt dabei empfindlich von der Anregungsenergie ab und beträgt $\approx 7$ für $\mathrm{E}_{\text {int }}=6.8 \mathrm{eV}$ und $\approx 0.1$ für $\mathrm{E}_{\text {int }}=5.1 \mathrm{eV}^{21}$ 
Im Experiment kommt es ab einer Stoßenergie von circa $\mathrm{E}_{\mathrm{cm}}=2.0 \mathrm{eV}$ (bzw. $\mathrm{E}_{\mathrm{int}}=$ $\left.\mathrm{E}_{\text {int }}^{298 K}\left(\mathrm{Ge}_{9} \mathrm{R}_{3}{ }^{-}\right)+\mathrm{E}_{\mathrm{cm}}=4.7 \mathrm{eV}\right)$ zur Bildung von $\mathrm{Ge}_{9} \mathrm{R}^{-}$, wobei die Bildung von $\mathrm{Ge}_{9} \mathrm{R}_{2}{ }^{-}$nur in Spuren erfolgt. Unser Modell sagt für $\mathrm{E}_{\text {int }}=4.7 \mathrm{eV}$ ein Verhältnis von $\left[\mathrm{Ge}_{9} \mathrm{R}^{-}\right] /\left[\mathrm{Ge}_{9} \mathrm{R}_{2}{ }^{-}\right] \approx 50$ voraus, was dem experimentellen Befund entspricht. ${ }^{22}$ Ein alternativer Bildungsweg von $\mathrm{Ge}_{9} \mathrm{R}^{-}$wäre die zweimalige, konsekutive Abspaltung von $\mathrm{R}^{\bullet}$ aus $\mathrm{Ge}_{9} \mathrm{R}_{3}^{-}$. Dieser Weg kann jedoch ausgeschlossen werden, da ein hypothetisches $\mathrm{Ge}_{9} \mathrm{R}_{2}{ }^{--}$Teilchen, welches ausgehend von $\mathrm{Ge}_{9} \mathrm{R}_{3}^{-}$(bei $\mathrm{E}_{\text {int }}=4.7 \mathrm{eV}$ ) gebildet wird, bezüglich des Zerfalls zu $\mathrm{Ge}_{9} \mathrm{R}^{-}+\mathrm{R}^{\bullet}$, stabil wäre. Mit einer berechnet Dissoziationsenergie von $1.49 \mathrm{eV}$, werden formal Lebensdauern im Bereich von $10^{16}$ Sekunden (ca. 300 Mio. Jahre) vorausgesagt. Das bedeutet, dass die Bildung von $\mathrm{Ge}_{9} \mathrm{R}^{-}$über ein kurzlebiges Intermediat $\left[\mathrm{Ge}_{9} \mathrm{R}_{2}\right]^{\bullet * *}$ sicher ausgeschlossen werden kann. Die Bildung von $\mathrm{Ge}_{9} \mathrm{R}^{-}$aus 1 scheint somit tatsächlich unter der Abspaltung von $\mathrm{Si}_{2}\left(\mathrm{SiMe}_{3}\right)_{6}$ in einem Schritt abzulaufen! $!^{23}$

\section{B.) Gasphasenreaktion von $\left[\mathrm{Ge}_{2} \underline{R}_{3}\right]^{-1} 1$ mit $\mathrm{O}_{2} \underline{\text { und } \mathrm{Cl}_{2}}$}

\section{$\underline{\mathbf{O}}_{2}:$}

Setzt man die in der Penning-Falle des FT-ICR-Massenspektrometers isolierten Anionen $\left[\mathrm{Ge}_{9} \mathrm{R}_{3}\right]^{-} 1$ einer Sauerstoffatmosphäre bei ca. $10^{-6}$ mbar aus, so beobachtet man keine Reaktion, d.h. es werden keine anionischen Produktspezies detektiert. ${ }^{24}$ Dies zeigt, dass 1 in der Gasphase unter den gegebenen experimentellen Bedingungen gegenüber Sauerstoff stabil ist. Ein vergleichbares Verhalten wurde schon bei nackten $\mathrm{Al}_{13}{ }^{-}$Clustern mit gerader Elektronenzahl beobachtet, ${ }^{25}$ wobei das inerte Verhalten dort auf das Vorliegen eines singulett Grundzustandes zurückgeführt wurde. Da auch 1 in einem Singulettgrundzustand vorliegt, könnte die Reaktionsträgheit von 1 gegenüber $\mathrm{O}_{2}$ ebenfalls auf einen spinverbotenen Übergangszustand zurückführbar sein.

\section{$\mathrm{Cl}_{2}:$}


Da sich bei Untersuchungen zur Gasphasenreaktivität von nackten Aluminiumclustern gezeigt hat, dass auch geschlossenschalige Cluster mit elementarem Chlor reagieren, ${ }^{26}$ wurden im Folgenden Versuche zum oxidativen Abbau von 1 mit elementarem Chlor durchgeführt. Setzt man 1 einer Chloratmosphäre von ca. $10^{-8}$ mbar aus, so beobachtet man nach einer Reaktionszeit von einigen Sekunden eine Vielzahl von Produktspezies des oxidativen Abbaus. Ein repräsentatives Massenspektrum zeigt Abbildung 3, wobei den einzelnen Massenpeaks verschiedene, dort angegebene Gasphasenspezies zugeordnet sind. Die zugewiesenen Summenformeln wurden durch einen Vergleich der gemessenen Isotopenverteilung der Massenpeaks mit den berechneten Isotopenverteilungen verifiziert (siehe Abbildungen Z2a und $\mathrm{Z} 2 \mathrm{~b}$ in den Zusatzinformationen).

Bei der Gasphasenreaktion von $\left[\mathrm{Ge}_{9} \mathrm{R}_{3}\right]^{-} \mathbf{1}$ mit elementarem Chlor bilden sich unter anderem die Clusterspezies $\left[\mathrm{Ge}_{9} \mathrm{R}_{3} \mathrm{Cl}_{2}\right]^{-} \mathbf{1 C l}_{\mathbf{2}}$ und $\left[\mathrm{Ge}_{9} \mathrm{R}_{3} \mathrm{Cl}_{4}\right]^{-} \mathbf{1} \mathbf{C l}_{\mathbf{4}}$, bei denen ein bzw. zwei Chlormoleküle oxidativ an $\mathbf{1}$ addiert wurden. Des Weiteren sind Abbauprodukte mit neun, acht und sieben Germaniumatomen zu erkennen: $\left[\mathrm{Ge}_{9} \mathrm{R}_{2} \mathrm{Cl}\right]^{-}$6; $\left[\mathrm{Ge}_{9} \mathrm{RCl}_{2}\right]^{-}$7, $\left[\mathrm{Ge}_{9} \mathrm{R}\right]^{-} \mathbf{2}$, $\left[\mathrm{Ge}_{9} \mathrm{Cl}\right]^{-} \mathbf{8},\left[\mathrm{Ge}_{9}\left\{\mathrm{Si}_{11} \mathrm{Me}_{24}\right\} \mathrm{Cl}\right]^{-} \mathbf{9},\left[\mathrm{Ge}_{9}\left\{\mathrm{Si}_{10} \mathrm{Me}_{21}\right\} \mathrm{Cl}_{2}\right]^{-} \mathbf{1 0},\left[\mathrm{Ge}_{8} \mathrm{R}\right]^{-} 11$ und $\left[\mathrm{Ge}_{7} \mathrm{R}\right]^{-} 12$.

$<<$ Abbildung 3 hier $>>>$

\begin{abstract}
Abbildung 3: Massenspektrum von $\left[\mathrm{Ge}_{9} \mathrm{R}_{3}\right]^{-} \mathbf{1}$ nach einer Reaktionszeit von 15 Sekunden, bei einem Chlorpartialdruck von $7 \cdot 10^{-8}$ mbar. Den einzelnen Massenpeaks sind die entsprechenden Summenformeln zugeordnet.
\end{abstract}

Das Auftreten einer Vielzahl verschiedener Gasphasenspezies deutet auf ein komplexes Reaktionsgeschehen hin, wobei zunächst die Frage zu klären ist, ob man es mit Parallel- oder Folgereaktionen $\mathrm{zu}$ tun hat. Dazu wurden Massenspektren nach unterschiedlichen Reaktionszeiten aufgenommen und die normierten Ionensignale von 1, sowie die der Produkte 
als Funktion der Reaktionszeit aufgetragen (Abbildung 4). Dabei zeigt sich als erstes, dass die Konzentration der Hauptprodukte $\left[\mathrm{Ge}_{9} \mathrm{R}_{3} \mathrm{Cl}_{2}\right]^{-} \mathbf{1 C l}_{2},\left[\mathrm{Ge}_{9} \mathrm{R}_{2} \mathrm{Cl}\right]^{-} \mathbf{6}$ und $\left[\mathrm{Ge}_{9}\left\{\mathrm{Si}_{11} \mathrm{Me}_{24}\right\} \mathrm{Cl}\right]^{-} \mathbf{9}$ gleichzeitig zunimmt, während die Konzentration von $\mathbf{1}$ einfach exponentiell abnimmt. $\mathbf{1 C l}_{\mathbf{2}}$ kann somit keine notwendige Zwischenstufe bei der Bildung von 6 bzw. von 9 sein. Zur weiteren experimentellen Bestätigung dieses Befundes wurde während der Reaktionszeit von $\mathbf{1}$ mit Chlor die Produktspezies $\mathbf{1} \mathbf{C l}_{\mathbf{2}}$ kontinuierlich aus der ICR-Zelle über eine resonante dipolare Anregung entfernt. Wäre $\mathbf{1} \mathbf{C l}_{\mathbf{2}}$ eine notwendige Zwischenstufe für die Bildung von $\mathbf{6}$ und 9, so sollte nun weder 6 noch 9 gebildet werden. Da der Konzentrationsverlauf von $\left[\mathrm{Ge}_{9} \mathrm{R}_{2} \mathrm{Cl}\right]^{-} \mathbf{6}$ und $\left[\mathrm{Ge}_{9}\left\{\mathrm{Si}_{11} \mathrm{Me}_{24}\right\} \mathrm{Cl}\right]^{-} \mathbf{9}$ nach Eliminierung von $\mathbf{1 C l}_{\mathbf{2}}$ nahezu unbeeinflusst ist, kann man $\mathbf{1 C l}_{2}$ als Zwischenstufe für die Bildung von $\mathbf{6}$ bzw. 9 ausschließen.

Ausgehend von $\mathbf{1 C l}_{\mathbf{2}}$ kommt man durch eine weitere Addition von $\mathrm{Cl}_{2} \mathrm{zu} \mathbf{1} \mathbf{C l}_{\mathbf{4}}$, was sich am gekoppelten Konzentrationsverlauf von $\mathbf{1} \mathbf{C l}_{\mathbf{2}}$ und $\mathbf{1} \mathbf{C l}_{\mathbf{4}}$ erkennen lässt.

$<<$ Abbildung 4 hier $>>>$

\begin{abstract}
Abbildung 4: Semilogarithmische Darstellung des zeitlichen Verlaufs der Konzentration der verschiedenen Gasphasenspezies $\quad\left[\mathrm{Ge}_{9} \mathrm{R}_{3} \mathrm{Cl}_{2}\right]^{-} \quad \mathbf{1 C l}_{\mathbf{2}}, \quad\left[\mathrm{Ge}_{9} \mathrm{R}_{3} \mathrm{Cl}_{4}\right]^{-} \quad \mathbf{1 C l}_{\mathbf{4}}, \quad\left[\mathrm{Ge}_{9} \mathrm{R}_{2} \mathrm{Cl}\right]^{-} \mathbf{6}$, $\left[\mathrm{Ge}_{9} \mathrm{RCl}_{2}\right]^{-}$7, $\left[\mathrm{Ge}_{9} \mathrm{R}\right]^{-}$2, $\left[\mathrm{Ge}_{9}\left\{\mathrm{Si}_{11} \mathrm{Me}_{24}\right\} \mathrm{Cl}\right]^{-} 9$ und $\left[\mathrm{Ge}_{9}\left\{\mathrm{Si}_{10} \mathrm{Me}_{21}\right\} \mathrm{Cl}_{2}\right]^{-} \mathbf{1 0}$ während der Gasphasenreaktion von $\left[\mathrm{Ge}_{9} \mathrm{R}_{3}\right]^{-} \quad \mathbf{1}$ mit $\mathrm{Cl}_{2}$ (der Übersichtlichkeit halber wurden Gasphasenspezies mit geringer Konzentration wie $\left[\mathrm{Ge}_{9} \mathrm{Cl}\right]^{-} 8$ nicht dargestellt). Die durchgezogenen Kurven resultieren aus einer Kurvenanpassung der gemessenen Datenpunkte (siehe Zusatzinformationen Abbildung Z4 und Tabelle Z1).
\end{abstract}

Die Bildung der primären Abbauprodukte mit neun Germaniumatomen $(\mathbf{6 , 7 , 9}$ und 10) lassen sich nach den Reaktionsgleichungen (1) - (4) plausibel machen: 


$$
\begin{aligned}
& {\left[\mathrm{Ge}_{9} \mathrm{R}_{3}\right]^{-} \mathbf{1}+\mathrm{Cl}_{2} \rightarrow\left[\mathrm{Ge}_{9} \mathrm{R}_{3} \mathrm{Cl}_{2}\right]^{-} \mathbf{1 C l} \mathbf{C}^{`} \rightarrow\left[\mathrm{Ge}_{9} \mathrm{R}_{2} \mathrm{Cl}\right]^{-} \mathbf{6}+\mathrm{R}-\mathrm{Cl}} \\
& {\left[\mathrm{Ge}_{9} \mathrm{R}_{2} \mathrm{Cl}\right]^{-} \mathbf{6}+\mathrm{Cl}_{2} \rightarrow\left[\mathrm{Ge}_{9} \mathrm{R}_{2} \mathrm{Cl}_{3}\right]^{-} \mathbf{6} \rightarrow\left[\mathrm{Ge}_{9} \mathrm{RCl}_{2}\right]^{-} \mathbf{7}+\mathrm{R}-\mathrm{Cl}} \\
& {\left[\mathrm{Ge}_{9} \mathrm{R}_{3}\right]^{-} \mathbf{1}+\mathrm{Cl}_{2} \rightarrow\left[\mathrm{Ge}_{9} \mathrm{R}_{3} \mathrm{Cl}_{2}\right]^{-} \mathbf{1 C l}{ }^{\prime}{ }^{\prime} \rightarrow\left[\mathrm{Ge}_{9}\left\{\mathrm{Si}_{11} \mathrm{Me}_{24}\right\} \mathrm{Cl}\right]^{-} \mathbf{9}+\mathrm{Cl}-\mathrm{SiMe}_{3}} \\
& \mathbf{9}+\mathrm{Cl}_{2} \rightarrow\left[\mathrm{Ge}_{9}\left\{\mathrm{Si}_{11} \mathrm{Me}_{24}\right\} \mathrm{Cl}_{3}\right]^{-} \mathbf{9}^{`} \rightarrow\left[\mathrm{Ge}_{9}\left\{\mathrm{Si}_{10} \mathrm{Me}_{21}\right\} \mathrm{Cl}_{2}\right]^{-} \mathbf{1 0}+\mathrm{Cl}-\mathrm{SiMe}_{3}
\end{aligned}
$$

Bei der Bildung von 6 und 7 wird ein $\mathrm{Si}\left(\mathrm{SiMe}_{3}\right)_{3}$ Ligand komplett als $\mathrm{Cl}-\mathrm{Si}\left(\mathrm{SiMe}_{3}\right)_{3}$ abgespalten, wobei ein $\mathrm{Cl}$ Atom am Cluster verbleibt. Bei den Produktspezies 9 und 10 kommt es zum oxidativen Abbau des Liganden unter Eliminierung von $\mathrm{Cl}-\mathrm{SiMe}_{3},{ }^{27}$ wobei der Ligand durch Spaltung einer Si-Si Bindung abgebaut wird. Dieser Reaktionsverlauf ist zu erwarten, da auch der oxidative Abbau von $\mathrm{Cl}-\mathrm{Si}\left(\mathrm{SiMe}_{3}\right)_{3}$ mit elementarem Chlor in Lösung, unter Spaltung einer Si-Si Bindung, $\mathrm{zu} \mathrm{Cl}_{2} \mathrm{Si}\left(\mathrm{SiMe}_{3}\right)_{2}$ führt. $^{28}$

Bei den Reaktionsgleichungen (1) und (3) haben die primären Oxidationsprodukte $\mathbf{1} \mathbf{C l}_{\mathbf{2}}$ ` und $\mathbf{1 C l}_{\mathbf{2}}$ " die gleiche Summenformel wie $\mathbf{1 C l}_{\mathbf{2}}$. Berücksichtigt man weiterhin, dass $\mathbf{1} \mathbf{C l}_{\mathbf{2}}$ wie oben erwähnt, keine Zwischenstufe auf dem Weg zu 6 bzw. 9 ist, so müssen die primär gebildeten Oxidationsprodukte $\mathbf{1 C l}_{\mathbf{2}}$ und $\mathbf{1 C l}_{\mathbf{2}}{ }^{\prime \prime}$ Isomere von $\mathbf{1 C l}_{\mathbf{2}}$ sein, die eine so geringe Lebensdauer aufweisen, dass sie nicht detektierbar sind. Solche im FT-ICR Massenspektrometer nicht detektierbaren Zwischenstufen wurden schon bei Gasphasenuntersuchungen des oxidativen Abbaus von Aluminiumclustern mit elementarem Chlor beschrieben. Dort hatten die primären Oxidationsprodukte eine Lebensdauer im Bereich von Nanosekunden und waren deshalb nicht detektierbar. ${ }^{26}$

Im Gegensatz zu den Untersuchungen bei „,nackten“ Aluminiumclustern werden bei der Oxidation von $\left[\mathrm{Ge}_{9} \mathrm{R}_{3}\right]^{-} \mathbf{1}$ mit elementarem Chlor somit zusätzlich detektierbare Oxidationsprodukte $\mathbf{1} \mathbf{C l}_{\mathbf{2}}$ und $\mathbf{1 C l}_{\mathbf{4}}$ gebildet. Zusammenfassend werden die hier identifizierten primären Schritte der Gasphasenreaktion von $\left[\mathrm{Ge}_{9} \mathrm{R}_{3}\right]^{-} \mathbf{1}$ mit $\mathrm{Cl}_{2}$ in Abbildung 5 wiedergegeben. 


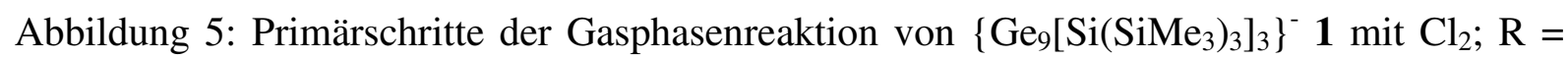
$\mathrm{Si}\left(\mathrm{SiMe}_{3}\right)_{3}$.

Die Bildung unterschiedlich stabiler, isomerer Primärprodukte $\mathbf{1 C l}_{\mathbf{2}}, \mathbf{1 C l}_{\mathbf{2}} \mathbf{2}^{`}$ und $\mathbf{1 C l}_{\mathbf{2}}{ }^{\prime}$ deutet daraufhin, dass ein $\mathrm{Cl}_{2}$ Molekül an verschiedenen Orten von 1 angreift, wobei sowohl eine Oxidation am Liganden als auch am Germaniumkern stattfindet. Im Folgenden soll der Angriff am Germaniumkern näher betrachtet werden:

Dazu wurden verschiedene Isomere von $\mathbf{1 C l}_{\mathbf{2}}$ mit Hilfe quantenchemischer Methoden berechnet, wobei angenommen wird, dass der Angriff von $\mathrm{Cl}_{2}$ an den „nackten“ Germaniumatomen bevorzugt ist. ${ }^{29}$ Ein solcher Angriff erscheint plausibel, da auch bei der Oxidation einer Ge(111) Oberfläche die „ungesättigten“ Germaniumatome der Oberfläche unter Bildung von Ge-Cl Einfachbindungen oxidiert werden. ${ }^{30}$ Aufgrund der sechs nackten Germaniumatome in $\mathbf{1}$ ergeben sich bei der Reaktion mit $\mathrm{Cl}_{2}$ eine Vielzahl möglicher Produkte, wobei zu erwarten ist, dass die beiden Chloratome im Produkt in räumlicher Nähe zueinander angeordnet sind. Die beiden mit DFT-Methoden ${ }^{17}$ berechneten Minimumstrukturen $\mathbf{1 C l}_{\mathbf{2}} \mathbf{a}$ bzw. $\mathbf{1 C l}_{\mathbf{2}} \mathbf{b}$, bei denen die beiden Chlorsubstituenten entweder an zwei unterschiedliche oder an dasselbe Germaniumatom gebunden sind, sind in Abbildung 6 wiedergegeben. 
$<<$ Abbildung 6 hier $>>>$

\begin{abstract}
Abbildung 6: Berechnete Molekülstruktur zweier Isomere des Primärproduktes $\left[\mathrm{Ge}_{9} \mathrm{R}_{3} \mathrm{Cl}_{2}\right]^{-}$ $\mathbf{1 C l}_{2}$ der Gasphasenreaktion von $\left[\mathrm{Ge}_{9} \mathrm{R}_{3}\right]^{-} \mathbf{1}$ mit $\mathrm{Cl}_{2}$ (die $\mathrm{SiMe}_{3}$ Gruppen sind der Übersichtlichkeit halber nicht dargestellt; $\left.\mathrm{R}=\mathrm{Si}\left(\mathrm{SiMe}_{3}\right)_{3}\right)$ : links: $\mathbf{1 C l}_{\mathbf{2}} \mathbf{a}$; rechts $\mathbf{1 C l}_{\mathbf{2}} \mathbf{b}$.
\end{abstract}

Bei der Bildung von $\mathbf{1 C l}_{2} \mathbf{a}$ aus $\mathbf{1}$ und $\mathrm{Cl}_{2}$ werden $310 \mathrm{~kJ} / \mathrm{mol}$ Energie frei und bei der Bildung des Isomers $\mathbf{1 C l}_{\mathbf{2}} \mathbf{b} 295 \mathrm{~kJ} / \mathrm{mol}$, d.h. das Isomer $\mathbf{1 C l}_{\mathbf{2}} \mathbf{a}$ ist um $15 \mathrm{~kJ} / \mathrm{mol}$ stabiler als $\mathbf{1 C l}_{\mathbf{2}} \mathbf{b}$. Bei den berechneten Molekülstrukturen von $\mathbf{1 C l}_{\mathbf{2}} \mathbf{a}$ und $\mathbf{1 C l}_{\mathbf{2}} \mathbf{b}$ fällt zunächst auf, dass der polyedrische Clusterkern durch die Oxidation aufgebrochen wird. Ein vergleichbares strukturelles Verhalten findet man auch bei den vollständig substituierten Clusterverbindungen des Germaniums: So wird die polycyclische $\mathrm{Ge}_{8} \mathrm{R}_{8} \mathrm{X}_{2}$ Verbindung $(\mathrm{R}=$ ${ }^{t} \mathrm{Bu}, \mathrm{X}=\mathrm{Br} ;{ }^{31} \mathrm{X}=\mathrm{Cl}^{32}$ ) als eine plausible Vorstufe zur geschlossenen würfelförmigen $\mathrm{Ge}_{8} \mathrm{R}_{8}$ Clusterverbindung $\left(\mathrm{R}=2,6-\mathrm{Me}_{2}-\mathrm{C}_{6} \mathrm{H}_{3},{ }^{33} \mathrm{R}=1\right.$-ethyl-1-methylpropyl ${ }^{34}$ ) diskutiert. ${ }^{35}$

Die Chlorsubstituenten in $\mathbf{1 C l}_{\mathbf{2}} \mathbf{a}$ sind terminal mit einem mittleren Ge-Cl Abstand von 233.6 pm gebunden und in $\mathbf{1 C l}_{\mathbf{2}} \mathbf{b}$ wird ein etwas kürzerer $\mathrm{Ge}-\mathrm{Cl}$ Abstand von 230.6 pm berechnet. Die berechneten $\mathrm{Ge}-\mathrm{Cl}$ Abstände sind dabei nur geringfügig länger als der $\mathrm{Ge}-\mathrm{Cl}$ Einfachbindungsabstand in $\mathrm{GeCl}_{2} \cdot$ Dioxan mit $228.1 \mathrm{pm} .{ }^{36}$

Betrachtet man die elektronische Situation in den beiden berechneten primären Oxidationsprodukten $\mathbf{1 C l}_{\mathbf{2}} \mathbf{a}$ und $\mathbf{1 C l}_{\mathbf{2}} \mathbf{b}$ mit Hilfe einer Ahlrichs-Heinzmann Populationsanalyse, so werden im Bereich der oxidierten Germaniumatome Dreizentrenbindungsanteile mit einer SEN (shared electron number) von maximal 0.17 berechnet. Bei den Dreizentrenbindungsanteilen in $\mathbf{1}$ wurde demgegenüber eine maximale SEN von 0.32 erhalten. Somit hat die Oxidation primär die delokalisierte Bindungssituation im Clustergerüst von 1 deutlich gestört. 
Dieser Befund zeigt die Besonderheit von 1 im Vergleich zu einer anderen großen Gruppe an Clusterverbindungen der 14. Gruppe, den Zintl-Ionen. Bei den Zintl-Ionen führt eine primäre Oxidation von z.B. $\mathrm{Ge}_{9}{ }^{4-}$ zur Bildung von Oligomeren $\left(\mathrm{Ge}_{9}\right)_{\mathrm{n}}{ }^{\mathrm{x}-}{ }^{37}$ bei denen die $\mathrm{Ge}_{9}$ Einheiten nur leicht verzerrt sind. Die Oxidation kann dabei auch zu neutralen „Polymeren“, d.h. neuartigen Festkörpermodifikationen führen, in denen die $\mathrm{Ge}_{9}$ Einheiten immer noch vorhanden sind. ${ }^{38}$ Demgegenüber wird durch die Oxidation der metalloiden Clusterverbindung $\left[\mathrm{Ge}_{9} \mathrm{R}_{3}\right]^{-} \mathbf{1}$ die delokalisierte Bindungssituation aufgehoben, d.h. der Cluster wird abgebaut, wobei neben den drei Liganden zusätzlich bis zu vier Chloratome in $\mathbf{1 C l}_{4}$ gebunden sind.

Ausgehend von einem der beiden Isomeren $\mathbf{1 C l}_{\mathbf{2}} \mathbf{a}$ oder $\mathbf{1 C l}_{\mathbf{2}} \mathbf{b}$ kommt es zur Abbaureaktion, bei der $\left(\mathrm{SiMe}_{3}\right)_{3} \mathrm{Si}-\mathrm{Cl}$ eliminiert und die Produktspezies $\left[\mathrm{Ge}_{9} \mathrm{R}_{2} \mathrm{Cl}\right]^{-} \mathbf{6}$ erhalten wird, deren berechnete Minimumstruktur in den Zusatzinformationen $\mathrm{zu}$ finden ist (Abbildung Z5). Ausgehend vom Isomer $\mathbf{1 C l}_{\mathbf{2}} \mathbf{b}$ erscheint die Eliminierung der vorgebildeten Hochtemperaturspezies $\mathrm{GeCl}_{2}$ am plausibelsten. Dafür müssten jedoch zwei Ge-Ge Bindungen gebrochen werden, deren Bindungslänge mit 249.7 und 257.2 pm im Bereich einer normalen Ge-Ge Einfachbindung von z.B. 245 pm wie in $\alpha$-Germanium ${ }^{39}$ liegen. Die durch die Chlorierung frei gewordene, berechnete Reaktionsenergie von $295 \mathrm{~kJ} / \mathrm{mol}$ ist jedoch deutlich kleiner als die doppelte Bindungsenergie einer Ge-Ge Einfachbindung von insgesamt ca. $527 \mathrm{~kJ} / \mathrm{mol}(2 \cdot 263.5 \pm 7 \mathrm{~kJ} / \mathrm{mol}),{ }^{40}$ so dass $\mathbf{1 C l}_{\mathbf{2}} \mathbf{b}$ möglicherweise das beobachtete stabile Oxidationsprodukt ist.

Als Ausgangsverbindung für den weiteren Abbau wird im Folgenden somit das Isomer $\mathbf{1 C l}_{\mathbf{2}} \mathbf{a}$ betrachtet. Nach quantenchemischen Rechnungen ist ausgehend von $\mathbf{1 C l}_{\mathbf{2}} \mathbf{a}$ die Bildung von $\mathbf{6}$ mit $20.3 \mathrm{~kJ} / \mathrm{mol}$ endotherm. Da bei der primären Oxidationsreaktion $310 \mathrm{~kJ} / \mathrm{mol}$ frei werden, ist die Bildung von 6 durch Reaktion von $\left[\mathrm{Ge}_{9} \mathrm{R}_{3}\right]^{-} \mathbf{1}$ mit $\mathrm{Cl}_{2}$ mit insgesamt ca. $290 \mathrm{~kJ} / \mathrm{mol}$ exotherm. 


\section{Zusammenfassung und Ausblick}

Das im Kristall strukturell charakterisierte Clusteranion $\mathrm{Ge}_{9} \mathrm{R}_{3}{ }^{-} \mathbf{1}\left(\mathrm{R}=\mathrm{Si}\left(\mathrm{SiMe}_{3}\right)_{3}\right)$ lässt sich über die ESI-Methode unzersetzt in die Gasphase überführen und in einem FTMassenspektrometer hinsichtlich seiner Fragmentierung und seines oxidativen Abbaus untersuchen. Bei der Fragmentierung von angeregten Clustermolekülen 1 zeigt sich, dass im ersten Schritt eine konzertierte Reaktion abläuft, bei der letztendlich ein $R_{2}$ Molekül abgespalten wird. Dieser anschaulich nicht leicht zu verstehende Prozess der Bildung und Abspaltung von sterisch überfrachteten $\mathrm{Si}_{2}\left(\mathrm{SiMe}_{3}\right)_{6}$ Molekülen, der an viele andere reduktive Eliminierungen (z.B. $\mathrm{C}_{2} \mathrm{H}_{6} \rightarrow \mathrm{C}_{2} \mathrm{H}_{4}+\mathrm{H}_{2}$ ) erinnert, konnte sowohl experimentell, durch die Bestimmung der erforderlichen Schwellenenergien, als auch durch DFT Rechnungen und durch Berechnung der Lebensdauer der beteiligten Spezies unter den eingestellten Fragmentierungsbedingungen bestätigt werden. Die Aufklärung einer solchen reduktiven Eliminierung an der „Oberfläche“ eines metalloiden Clusters dürfte auch von genereller Bedeutung sein, da hier z.B. ein Modellsystem für Oberflächenreaktionen in der heterogenen Katalyse vorliegt. Die vollständige Dissoziation aller Liganden R führt zum „,nackten“ $\mathrm{Ge}_{9}{ }^{\text {, }}$ d.h. durch reduktive Eliminierung wechselt man von der Seite der metalloiden Cluster (mittlere positive Oxidationszahl der Germaniumatome) in den Bereich der Zintl-Ionen (mittlere negative Oxidationszahl der Germaniumatome).

Orientierende Oxidationsversuche von $\mathbf{1}$ mit elementarem Sauerstoff zeigen, dass $\mathbf{1}$ in der Gasphase gegenüber $\mathrm{O}_{2}$ inert ist, d.h. die primäre Oxidation ist durch einen spinverbotenen Übergangszustand analog zu jüngsten Oxidationsversuchen von $\mathrm{Al}_{13}{ }^{-}$Clusteranionen nicht möglich. Dies steht nicht im Widerspruch zum pyrophoren Verhalten von Kristallen von 1 gegenüber $\mathrm{O}_{2}$ : Offensichtlich wird das Spinverbot durch Wechselwirkung mit der Oberfläche aufgehoben, und die stark exotherme Oxidation kann stattfinden. Geplante Untersuchungen mit verschiedenen $\mathrm{Ge}_{\mathrm{n}}$-Clusterionen und $\mathrm{O}_{2}$ sollen zeigen, ob dem „Spinverbot“ bei 
Reaktionen zwischen isolierten Molekülen/Ionen tatsächlich die bei $\mathrm{Al}_{13}{ }^{-}$Reaktionen zuerst experimentell realisierte allgemeine Bedeutung zukommt.

Oxidationsexperimente von $\mathbf{1}$ mit $\mathrm{Cl}_{2}$ zeigen ein komplexes Reaktionsgeschehen, wobei drei primäre Reaktionskanäle beobachtet werden (Abbildung 5). So kommt es zum einen zur Bildung von stabilen Oxidationsprodukten $\mathbf{1 C l}_{\mathbf{2}}$ und $\mathbf{1} \mathbf{C l}_{\mathbf{4}}$ bzw. zu Abbauprodukten, bei denen entweder $\mathrm{Cl}-\mathrm{SiMe}_{3}(\mathbf{9}, \mathbf{1 0})$ oder $\mathrm{Cl}-\mathrm{Si}\left(\mathrm{SiMe}_{3}\right)_{3}(\mathbf{6}, \mathbf{7})$ abgespalten wird.

Erste orientierende theoretische Untersuchungen zum oxidativen $\mathrm{Cl}_{2}$ - $\mathrm{Abbau}$ von $\mathbf{1}$ deuten daraufhin, dass der Primärschritt beim Kontakt mit einem $\mathrm{Cl}_{2}$ Molekül entscheidend ist, d.h. die beiden Chloratome des $\mathrm{Cl}_{2}$ Moleküls können an ein oder an zwei Germaniumatome gebunden werden. Sämtliche bisher angenommenen vorläufigen Reaktionsschritte bei der Chlorierung von 1 sollen in zukünftigen weiterführenden Untersuchungen unter Einschluss von berechneten Übergangszuständen geklärt werden.

Alle bisher durchgeführten quantenchemischen Rechnungen zeigen allerdings eindeutig, dass die Oxidation von 1 mit $\mathrm{Cl}_{2}$ primär zum Verlust der delokalisierten Bindungssituation in $\mathbf{1}$ führt. Dies steht im Gegensatz zu Untersuchungen bei den Zintl-Ionen, bei denen in primären Oxidationsschritten die Bindungssituation innerhalb des Cluster nahezu unverändert bleibt. Dieser Befund ist zu erwarten, da die mittlere Oxidationsstufe bei den Zintl-Ionen negativ, bei den metalloiden Clustern demgegenüber positiv ist. Eine Oxidation führt bei den Zintl-Ionen also zuerst zum Element, bei den metalloiden Clustern demgegenüber „weiter weg“ vom Element hin $\mathrm{zu}$ oxidierten Spezies wie z.B. $\mathrm{GeCl}_{2}$ oder $\mathrm{GeCl}_{4}$. Die hier beobachtbaren Oxidationsreaktionen können somit als Elementarschritte für die Oxidation von elementarem Germanium mit Chlor verstanden werden, d.h. hier wird der Prozess der Chlorierung in Einzelschritten sichtbar.

Bei einer Reduktion dreht sich die Situation um, und man gelangt bei den metalloiden Clustern zum Element und bei den Zintl-Ionen entfernt man sich immer mehr vom Element, d.h. man erhält kleinere Clustereinheiten wie z.B. $\mathrm{Si}_{4}{ }^{4-}$ in $\mathrm{BaSi}_{2}{ }^{41}$ In der „Nähe“ des 
Elements, d.h. bei kleinen positiven als auch negativen mittleren Oxidationsstufen gleichen sich die beiden Verbindungsklassen strukturell an. So werden beispielsweise in beiden Typen von $\mathrm{E}_{9}$ Clustern die $\mathrm{zu}$ erwartenden Strukturen des einfach überdachten quadratischen Antiprismas bzw. des dreifach überdachten trigonal Prismas realisiert. Dieses Verhalten ist zu erwarten, da beide Clustertypen (metalloide Cluster und Zintl-Ionen) bei kleiner werdenden mittleren Oxidationsstufen (positiv oder negativ) demselben Endpunkt zustreben: dem Zustand des Elements. Gasphasenuntersuchungen zum Oxidationsverhalten von Zintl-Ionen fehlen bisher, könnten jedoch helfen die Zusammenhänge zwischen den beiden Klassen an Clusterverbindungen im Bereich der 14. Gruppe weiter zu präzisieren.

\section{Experimentelles}

Die massenspektrometrischen Gasphasenexperimente wurden an einem FT-ICRMassenspektrometer (IonSpec, Ultima) durchgeführt, welches mit einem $7 \mathrm{~T}$ Magneten (Cryomagnetics, Oak Ridge, TN, USA) ausgerüstet ist. Die Ionenerzeugung geschah mittels Elektrospray-Ionisierung (ESI) der gelösten Cluster 1 (ca. 0.01 mol/l) in Tetrahydrofuran (THF). Hierfür wurde eine kommerzielle ESI-Quelle (Analytica of Branford) verwendet, bei der der vordere Quellbereich durch eine selbstgebaute, evakuierbare Sprühkammer ersetzt wurde. $^{42}$ Die Lösung wurde mit einer Spritzenpumpe (Pumprate: $100 \mu \mathrm{l} / \mathrm{h}$ ) durch eine geerdete Edelstahlkapillare (Innendurchmesser: $100 \mu \mathrm{m}$ ) gepumpt. Das Potential der Endplatte und des Eingangs der Quarzkapillare betrug $3.2 \mathrm{kV}$ bzw. $3.3 \mathrm{kV}$. Am hinteren Ende der Quarzkapillare („Kapillarexit“) lag eine Spannung von -120 V an. Die Ionen gelangen dann durch den sich anschließenden Skimmer (ca. -20 V) in einen Hexapol, wo sie für 2-5 s zwischengespeichert bzw. akkumuliert wurden. Fragile Ionen fragmentieren u.U. schon infolge einer hohen Potentialdifferenz am Übergang von Quarzkapillare zu Skimmer. ${ }^{43}$ In 
diesem Fall kann die Fragmentierung unterbunden werden, wenn die „Kapillarexit“-Spannung auf ca. -30 V minimiert wird.

Der Ionentransfer in die ICR-Zelle geschieht mittels eines Quadrupols, in den das Ionenpaket aus dem Hexapol „eingepulst“ wird. Zur Speicherung von Anionen wurde typischerweise eine Speicherspannung von $-1.5 \mathrm{~V}$ verwendet. Die Ionenisolierung und Ionendetektion geschah mittels ICR-Standardtechniken. ${ }^{44}$

\section{CID-Experimente}

Zur Durchführung der CID- (collision induced dissociation) Experimente wurden die ClusterIonen zunächst wie oben beschrieben in die ICR-Zelle transferiert und $\mathrm{Ge}_{9} \mathrm{R}_{3}{ }^{-} \mathbf{1}$ wurde über eine SWIFT-Anregung ${ }^{16}$ (stored waveform inverse Fourier transform) isoliert. Auf eine Thermalisierung mit z.B. Helium oder Argon als Stoßgas wurde verzichtet, da hierdurch kein Einfluss auf die Fragmentierung zu beobachten war.

Nach der Isolierung wurden die Ionen entsprechend ihrer reduzierten Zyklotronfrequenz resonant dipolar angeregt und mit Argon als Stoßgas zur Kollision gebracht. Im Gegensatz zu einer nicht-resonanten Anregung, wie sie bei SORI (sustained off-resonance irradiation collisional activation dissociation) Experimenten angewandt wird, ist durch die resonante Anregung die kinetische Energie der Ionen definierter einzustellen.

Die kinetische Energie nach resonanter Anregung ergibt sich durch Gleichung $(1)^{44,45}$

$$
E_{k i n}=\beta^{2} \frac{q^{2} V_{p p}{ }^{2} t^{2}}{8 m d^{2}}
$$

wobei $q$ die Ionenladung, $m$ die Ionenmasse, $V_{p p}$ der Spitze-Spitze-Wert der an den Sendelektroden anliegenden RF-Spannung, $t$ die Anregungsdauer, $d$ der Durchmesser der ICR-Zelle (hier: $62.5 \mathrm{~mm}$ ) und $\beta$ ein von der Zellgeometrie abhängiger Geometriefaktor ist, 
der nicht genau bekannt ist, aber zu 0.80 - 0.89 abgeschätzt werden kann; ${ }^{46}$ im Folgenden wurde $\beta=0.89$ verwendet. Variation der kinetischen Energie erfolgte durch Änderung von $V_{p p}$ bei konstant gehaltener Anregungszeit $(t=500 \mu \mathrm{s})$. Die maximal zur Verfügung stehende Kollisionsenergie im Schwerpunktsystem $\left(E_{c m}\right)$, welche beim Stoß mit z.B. Argon in innere Energie umgewandelt werden kann, ergibt sich unter der Annahme stationärer Stoßgasatome gemäß Gleichung 2: ${ }^{47}$

$$
E_{c m}=E_{k i n} \frac{m_{g}}{m_{g}+m_{i o n}}
$$

wobei $m_{g}$ die Masse des Stoßgases und $m_{i o n}$ die Masse des angeregten Ions ist.

Für die CID-Experimente wurde Argon (99,9990 \%, Air Liquide) als Stoßgas über ein UHVLeckventil (Huntington) in die ICR-Zelle eingeleitet, womit ein statischer Argonpartialdruck von ca. $2 \cdot 10^{-8}$ mbar eingestellt (UHV-Basisdruck ca. $5 \cdot 10^{-10}$ mbar) wurde. Alle angegebenen Gasdrücke wurden bezüglich der Nachweisempfindlichkeit der Ionisations-Messröhre (Granville-Phillips) korrigiert. ${ }^{48,49}$

Um realistische Dissoziationsenergien aus den CID-Experimenten abzuleiten, ist es prinzipiell erforderlich, dass man möglichst unter Einzelstoßbedingungen arbeitet und den Anteil von Mehrfachstößen minimiert. ${ }^{50}$ Ein Druck von ca. $2 \cdot 10^{-8}$ mbar und eine Kollisionsdauer (= Zeitintervall zwischen Anregung der Ionen und ihrer Detektion) von 100 ms stellte sich als ideal heraus. Bei einer längeren Kollisionsdauer von 200 ms wurde eine Verschiebung der Schwellenergie hin zu kleineren Energiewerten festgestellt, was auf Mehrfachstöße zurïckzuführen ist. ${ }^{11}$

Im Vergleich zur Kollisionsdauer ist die Anregung für den Massennachweis mit $1 \mathrm{~ms}$ vernachlässigbar kurz. Falls Ionen während der Detektion stoßinduziert fragmentieren, sollten 
sie aufgrund des damit verbundenen Kohärenzverlustes nicht mehr effektiv nachgewiesen werden.

Für die Bestimmung der Dissoziationsschwellen wurden Massenspektren bei unterschiedlichen Kollisionsenergien aufgenommen, wobei pro Datenpunkt ca. 10 Spektren akkumuliert wurden. Als Maß für die Ionenintensität wurden die Signalamplituden aus den Massenspektren mit dem Programm DataView ${ }^{51}$ ausgelesen. Um einen möglichen Ionenverlust aus der ICR-Zelle zu kompensieren und um Signalinstabilitäten der Ionenquelle auszugleichen, wurden die Ionensignale $I$ auf die Summe aller detektierten Ionen $I_{0}$ (ursprünglich isoliertes Primärion und Fragmentionen) normiert. Die Schwellenergien für die Bildung der einzelnen Fragmente wurden durch eine geeignete Fitfunktion ermittelt, die u.a. die Dopplerverbreiterung des Stoßgases berücksichtigt. ${ }^{11,52}$

\section{Reaktionen mit Chlor}

Für die Reaktion von $\mathrm{Ge}_{9} \mathrm{R}_{3}{ }^{-}$mit Chlor wurde Chlorgas 2.8 (99.8\%) über das Leckventil in die ICR-Zelle eingeleitet. Es befand sich für die gesamte Dauer des Experimentes in der ICRZelle. Die Reaktion wurde im Druckbereich von ca. $4 \cdot 10^{-8}-9 \cdot 10^{-8}$ mbar untersucht. Nach dem Ionentransfer in die ICR-Zelle wurde eine Massenisolierung von $\mathrm{Ge}_{9} \mathrm{R}_{3}{ }^{-}$durchgeführt, worauf eine variable Reaktionszeit folgte, die mit dem Nachweis der Ionen endete. Eine Thermalisierung der Ionen war experimentell nicht durchführbar, da die Cluster 1 schon während der Thermalisierungszeit mit $\mathrm{Cl}_{2}$ reagiert hätten. Für die Messung von Reaktionskinetiken wurden Massenspektren nach unterschiedlichen Reaktionszeiten (= Zeitintervall zwischen Ionenisolierung und ihrer Detektion) aufgenommen und die normierten Ionensignale des Eduktes $\mathrm{Ge}_{9} \mathrm{R}_{3}{ }^{-}$sowie der Produkte wurden als Funktion der Reaktionszeit abgetragen. Typischerweise wurden 10 Spektren akkumuliert.

Um Geschwindigkeitskonstanten bezüglich der einzelnen Reaktionskanäle zu bestimmen, wurden mit dem Programm DetMech ${ }^{53}$ verschiedene Reaktionsmechanismen an die 
gemessenen Kinetiken angepasst, indem die Geschwindigkeits-Zeit-Gesetze mit Hilfe des Runge-Kutta-Verfahrens numerisch integriert wurden.

Um den Reaktionsmechanismus experimentell im Einzelnen aufzuklären, war es bei einigen Experimenten erforderlich, Reaktionsprodukte während der Reaktionszeit mit Chlor kontinuierlich aus der ICR-Zelle durch eine resonante, dipolare Anregung zu entfernen. ${ }^{54}$

\section{Literatur und Fußnoten}

${ }^{1}$ a.) K. Weiß, H. Schnöckel, Z. Anorg. Allg. Chem. 2003, 629, 1175 - 1183; b.) K. Koch, R. Burgert, H. Schnöckel, Angew. Chem. 2007, 119, 5897 - 5900; Angew. Chem. Int. Ed. 2007 46, 5795 - 5798; c.) K. Koch, H. Schnöckel, Z. Anorg. Allg. Chem. 2007, 633, 873 878.

${ }^{2}$ K. Koch, A. Schnepf, H. Schnöckel, Z. Anorg. All. Chem. 2006, 632, 1710 - 1716.

${ }^{3}$ J. W. Gauthier, T. R. Trautman, D. B. Jacobsen, Anal. Chim. Acta 1991, 246, 211-225

${ }^{4}$ A. Schnepf, Angew. Chem. 2004, 116, 680 - 682; Angew. Chem. Int. Ed. 2004, 43, 664 666; A. Schnepf, Coord. Chem. Rev. 2006, 250, 2758 - 2770.

${ }^{5}$ C. Schenk, A. Schnepf, Angew. Chem. 2007, 119, 5408 - 5410; Angew. Chem. Int. Ed. 2007 46, 5314 - 5316; C. Schenk, F. Henke, G. Santigo, I. Krossing, A. Schnepf, Dalton Trans. 2008, 33, 4436 - 4441; F. Henke, C. Schenk, A. Schnepf, Dalton Trans. 2009, 42, 9141 9145.

${ }^{6}$ C. Schenk, A. Schnepf, Chem. Commun. 2009, 22, 3208 - 3210.

${ }^{7}$ H. Schnöckel, Dalton Trans. 2005, 19, 3131 - 3136.

${ }^{8}$ A. Schnepf, Chem. Soc. Rev. 2007, 36, $745-758$.

${ }^{9}$ A. Schnepf, Angew. Chem. 2003, 115, 2728 - 2729; Angew. Chem. Int. Ed. 2003 42, 2624 2625. 
${ }^{10}$ J. B. Fenn, Angew. Chem. 2003, 115, 3999-4024; Angew. Chem. Int. Ed. 2003, 42, 3871 3894.

${ }^{11}$ H. L. Sievers, H.-Fr. Grützmacher, P. Caravatti, Int. J. Mass Spectrom. Ion Processes 1996, 157/158, 233-247.

12 J. Laskin, M. Byrd, J. Futrell, Int. J. Mass Spectrom. 2000, 195/196, 285-302.

${ }^{13}$ Bei den früheren SORI CAD Untersuchungen ${ }^{2}$ wurde in geringem Maß auch die radikalische Spezies $\mathrm{Ge}_{9} \mathrm{Si}\left(\mathrm{SiMe}_{3}\right)_{2}{ }^{-\bullet} \mathbf{2 a}$ beobachtet, so dass dort noch nicht klar war ob $\mathbf{3}$ direkt aus 2 durch Eliminierung von $\mathrm{Si}_{2} \mathrm{Me}_{6}$ entsteht oder durch zweifache Eliminierung von $\mathrm{SiMe}_{3}$. Die hier durchgeführten Einzelstoßexperimente zeigen jedoch eindeutig, dass ausgehend von 2 ausschließlich $\mathrm{Si}_{2} \mathrm{Me}_{6}$ eliminiert wird, so dass die bei den SORI CAD Untersuchungen beobachtbare geringe Konzentration an 2a eher auf einen Reaktionskanal geringer Wahrscheinlichkeit hinweist als auf einen intermediären Charakter von 2a auf dem Weg zu 3.

${ }^{14}$ Im Prinzip wäre es auch denkbar, dass 4 bei der resonanten Anregung von 2 letztendlich durch Eliminierung eines Si-Atom zu 5 fragmentiert $\left(\mathrm{Ge}_{9} \mathrm{Si}^{-} \rightarrow \mathrm{Ge}_{9}{ }^{-}+\mathrm{Si}\right)$. Da jedoch nach DFT Rechnungen die Eliminierung eines Ge-Atoms $\left(\mathrm{Ge}_{9} \mathrm{Si}^{-} \rightarrow \mathrm{Ge}_{8} \mathrm{Si}^{-}+\mathrm{Ge}\right)$ mit ca. 28.9 $\mathrm{kJ} / \mathrm{mol}$ gegenüber einer Si-Atom Eliminierung begünstigt ist, sollten bei einem solchen Fragmentierungsmechanismus ausschließlich siliziumhaltige Clusteranionen beobachtet werden, die jedoch nicht detektiert werden.

${ }^{15}$ Die Erzeugung von 2 wurde dabei mit SORI Anregung durchgeführt, da im Gegensatz zu on-resonanter Anregung die Fragmentation mit SORI wesentlich effizienter verläuft, was zu höheren Ausbeuten der Fragmentionen führt. Nach der Bildung von 2 wurden alle Ionen außer 2 durch eine SWIFT ${ }^{16}$ Anregung aus der Ionenfalle entfernt.

${ }^{16}$ T. C. L. Wang, T. L. Ricca, A. G. Marshall, Anal. Chem. 1986, 58, 2935-2938. 
${ }^{17}$ Quantenchemische Rechnungen wurden mit der RI-DFT Version des Turbomole Programmpackets durchgeführt, wobei das Becke-Perdew 86 Funktional verwendet wurde.

Die Basissätze waren von SVP Qualität. Die elektronische Struktur der berechneten Verbindungen wurde mit Hilfe einer Ahlrichs-Heinzmann Populationsanalyse untersucht. Turbomole: O. Treutler and R. Ahlrichs, J. Chem. Phys. 1995, 102, 346 - 354; BP-86functional: J. P. Perdew, Phys. Rev. B 1986, 33, 8822 - 8824; A. D. Becke, Phys. Rev. A 1988, 38, 3098 - 3100; RI-DFT: K. Eichkorn, O. Treutler, H. Öhm, M. Häser, R. Ahlrichs, Chem. Phys. Lett. 1995, 240, 283 - 290; SVP: A. Schäfer, H. Horn, R. Ahlrichs, J. Chem. Phys. 1992, 97, 2571 - 2577; Ahlrichs-Heinzmann population analysis: E. R. Davidson, J. Chem. Phys. 1967, 46, 3320 - 3324; K. R. Roby, Mol. Phys. 1974, 27, 81 - 104; R.

Heinzmann, R. Ahlrichs, Theor. Chim. Acta 1976, 42, 33 - 45; C. Erhardt, R. Ahlrichs, Theor. Chim. Acta 1985, 68, $231-245$.

${ }^{18}$ C. Lifshitz, Eur. J. Mass Spectrom. 2002, 8 85-98.

19 T. Baer und W. L. Hase, Unimolecular Reaction Dynamics, Theory and Experiment, Oxford University Press, New York 1996.

${ }^{20}$ M. Olzmann, R. Burgert und H. Schnöckel, J. Chem. Phys. 2009, 131, 174304.

21 Die für die Berechnung benötigten Parameter wie Schwingungsfrequenzen und Bindungsenergien entstammen aus DFT-Rechnungen.

22 Allerdings sind die für diese Energie berechneten Geschwindigkeitskonstanten um Größenordnungen zu klein. Hier zeigt sich die Sensitivität des Modells gegenüber Unsicherheiten in den berechneten Energien und Schwingungsdaten der Moleküle. Für eine genauere Voraussage der energieabhängigen Änderung der Selektivität und der Absolutbeträge der Geschwindigkeitskonstanten müssen sicher Anharmonizitätseffekte 
und ggf. auftretende Reaktionsschwellen berücksichtigt werden. Eine genaue quantitative Analyse ist jedoch nicht Gegenstand der vorliegenden Arbeit.

${ }^{23}$ Auch die Tatsache, dass $\mathrm{Ge}_{9} \mathrm{R}^{-}$bereits bei einer Stoßenergie von $2.0 \mathrm{eV}\left(\mathrm{E}_{\text {int }}=4.7 \mathrm{eV}\right)$ gebildet wird, spricht gegen den Mechanismus über die Bildung von $\mathrm{Ge}_{9} \mathrm{R}_{2}{ }^{\circ}$. Für diesen Pfad wird im Rahmen der PST eine notwendige innere Mindestenergie von $\mathrm{E}_{\text {int. }}\left(\mathrm{Ge}_{9} \mathrm{R}_{3}{ }^{-}\right)=$ $6.8 \mathrm{eV}$ berechnet.

${ }^{24}$ Für die Reaktion von $\mathrm{Ge}_{9} \mathrm{R}_{3}{ }^{-} \mathbf{1}$ mit $\mathrm{O}_{2}$ kann aus den Experimenten ein oberer Grenzwert bezüglich der Reaktionskonstanten von $\mathrm{k} \leq 6 \cdot 10^{-12} \mathrm{~cm}^{3} \mathrm{~s}^{-1}$ abgeschätzt werden.

${ }^{25}$ R. Burgert, H. Schnöckel, A. Grubisic, X. Li, S. T. Stokes, G. F. Ganteför, B. Kiran, P. Jena, K. H. Bowen, SCIENCE, 2008, 319, 438 - 442.

${ }^{26}$ R. Burgert, H. Schnöckel, M. Olzmann, K. H. Bowen, Angew. Chem. 2006, 118, 1505 1508; Angew. Chem. Int. Ed. 2006, 45, 1476 - 1479.

${ }^{27}$ Ausgehend vom Isotopenmuster ist auch noch das Abbauprodukt $\left\{\mathrm{Ge}_{8}\left[\mathrm{Si}_{12} \mathrm{Me}_{26} \mathrm{CH}_{2} \mathrm{Cl}\right]\right\}^{-}$ 12a denkbar, das ausgehend von $\mathbf{1 C l}_{\mathbf{2}}$ durch Eliminierung von $\mathrm{HGeCl}$ entstehen könnte. Nach quantenchemischen Rechnungen wäre diese Reaktion jedoch mit 248 kJ/mol endotherm. Da ausgehend von $\mathbf{1 C l}_{2} `$ die Bildung von $\left[\mathrm{Ge}_{9}\left\{\mathrm{Si}_{11} \mathrm{Me}_{24}\right\} \mathrm{Cl}\right]^{-} \mathbf{9}$ nur 62.7 $\mathrm{kJ} / \mathrm{mol}$ endotherm und somit vergleichbar der Bildungsenthalpie von $\left[\mathrm{Ge}_{9} \mathrm{R}_{2} \mathrm{Cl}\right]^{-} \mathbf{6}$ mit $20.3 \mathrm{~kJ} / \mathrm{mol}$ ist, ist zu erwarten, dass es sich bei der beobachteten Verbindung um das Abbauprodukt des Liganden $\left\{\mathrm{Ge}_{9}\left[\mathrm{Si}_{11} \mathrm{Me}_{24}\right] \mathrm{Cl}\right\}^{-} 9$ handelt.

${ }^{28}$ H. Gilman, R. L. Harrell, J. Organomet. Chem. 1966, 5, 199 - 200.

${ }^{29}$ Der Angriff von $\mathrm{Cl}_{2}$ erfolgt somit nicht am $\mathrm{HOMO}$, dass laut quantechemischen Rechnungen an den ligandengebundenen Germaniumatomen lokalisiert ist. Diese Germaniumatome stehen jedoch aufgrund der Abschirmung durch die $\mathrm{Si}\left(\mathrm{SiMe}_{3}\right)_{3}$ 
Liganden nicht zur Verfügung, so dass ein Angriff an den nackten Germaniumatomen wahrscheinlicher ist.

${ }^{30}$ P. W. Loscutoff, S. F. Bent, Annu. Rev. Phys. Chem. 2006, 57, 467 - 495.

${ }^{31}$ M. Weidenbruch, F.-T. Grimm, S. Pohl, W. Saak, Angew. Chem. 1989, 101, 201; Angew. Chem. Int. Ed. 1989, 28, 198.

${ }^{32}$ A. Sekuguchi, H. Naito, H. Nameki, K. Ebata, C. Kabuto, H. Sakurai, J. Organomet. Chem. 1989, 368, C1 - C4.

${ }^{33}$ A. Sekiguchi, T. Yatabe, H. Kamatani, C. Kabuto, H. Sakurai, J. Am. Chem. Soc. 1992, $114,6260-6262$.

${ }^{34}$ M. Unno, K. Higuchi, K. Furuya, H. Shioyama, S. Kyushin, M. Goto, H. Matsumoto, Bull. Chem. Soc. Jpn. 2000, 73, 2093 - 2097.

35 A. Sekiguchi, H. Sakurai, Adv. Organomet. Chem. 1995, 37, 1 - 38.

${ }^{36}$ M. K. Denk, M. Kahn, A. L. Lough, K. Shuchi, Acta. Cryst. C, 1998, c54, 1830 - 1832.

${ }^{37}$ A. Ugrinov, S. Sevov, J. Am. Chem. Soc. 2002, 124, 10990 - 10991; A. Ugrinov, S. Sevov, Inorg. Chem. 2003, 42, 5789 - 5791; L. Yong, D. Hoffmann, T. F. Fässler, Z. Anorg. Allg. Chem. 2005, 631, $1149-1153$.

${ }^{38}$ G. S. Armatas, M. G. Kanatzidis, SCIENCE, 2006, 313, 817 - 820; G. S. Armatas, M. G. Kanatzidis, Nature, 2006, 441, 1122 - 1125; D. Sun, A. E. Riley, A. J. Cadby, E. K.

Richman, S. D. Korlann, S. H. Tolbert, Nature, 2006, 441, 1126 - 1130.

${ }^{39}$ Holleman-Wiberg, Lehrbuch der Anorganischen Chemie, 102. Auflage, Walter de Gruyter, Berlin, 2007, 1004.

${ }^{40}$ J. E. Kingcade, H.M. Nagarathna-Naik, I. Shim, K.A. Gingerich, J. Phys. Chem. 1986, 90, $2830-2834$. 
${ }^{41}$ H. Schäfer, K. H. Janzon, A. Weiß, Angew. Chem. 1963, 75, 451 - 452; Angew. Chem. Int. Ed. Engl. 1963, 2, $393-394$.

${ }^{42}$ Ralf Burgert, Dissertation, Universität Karlsruhe (TH), 2007.

${ }^{43}$ O. Hampe, M. Neumaier, M. N. Blom, M. M. Kappes, Chem. Phys. Lett. 2002, 354, 303309.

${ }^{44}$ A. G. Marshall, C. L. Hendrickson, G. S. Jackson, Mass Spectrom. Rev. 1998, 17, 1-35.

${ }^{45}$ A.G. Marshall, C.L. Hendrickson and G.S. Jackson. Encyclopedia of Analytical Chemistry 2000, 11694-11728.

${ }^{46}$ A. M. Hawkridge, A. I. Nepomuceno, S. L. Lovik, C. J. Mason, D. C. Muddiman, Rapid. Commun. Mass Spectrom. 2005, 19, 915 - 918.

${ }^{47}$ S. A. McLuckey, J. Am. Soc. Mass Spectrom. 1992, 3, 599-614.

${ }^{48}$ J. E. Bartmess, R. M Georgiadis, Vacuum 1983, 33, 149-153.

${ }^{49}$ M. L. Shaw, Rev. Sci. Instrum. 1966, 37, 113.

${ }^{50}$ C.E.C.A. Hop, T.B. McMahon, G.D. Willett, Int. J. Mass Spectrom. Ion Processes 1990, 101, 191-208.

${ }^{51}$ S. Gilb und D. Schooss. DataView, Version 2.2.22 2006.

52 J. P. Chantry, J. Chem. Phys. 1971, 55, 2746.

${ }^{53}$ E. Schumacher. DetMech, http://www.chemsoft.ch/chekin.htm 2003.

${ }^{54}$ Hiermit ist es z.B. möglich bei der hypothetischen Reaktion $\mathrm{A} \rightarrow \mathrm{B} \rightarrow \mathrm{C}$ das Produkt $\mathrm{B}$ als notwendige Vorstufe für die Bildung von $\mathrm{C}$ auszuschließen, falls $\mathrm{C}$ auch gebildet wird, wenn B kontinuierlich aus der ICR-Zelle entfernt wird, wobei das auch nur gilt, wenn die Anregung von B schneller erfolgt als die Reaktion von B nach C. Demnach muss die Bildung von $\mathrm{C}$ aus A erfolgen. 